OPEN ACCESS

Edited by:

Rujin Chen,

Lanzhou University, China

Reviewed by:

Stephan Pollmann,

National Institute of Agricultural and Food Research and Technology,

Spain

José López-Bucio,

Universidad Michoacana de San

Nicolás de Hidalgo, Mexico Katarzyna Retzer, Academy of Sciences of the Czech Republic, Czechia

*Correspondence:

Weiming Cai

wmcai@sibs.ac.cn,

wmcai@cemps.ac.cn

Qijun Xie

qjxie@hnu.edu.cn

Specialty section:

This article was submitted to

Plant Physiology,

a section of the journal

Frontiers in Plant Science

Received: 23 November 2020

Accepted: 15 February 2021

Published: 04 March 2021

Citation:

Xie Q, Essemine J, Pang X, Chen H, Jin J and Cai W (2021)

Abscisic Acid Regulates the Root Growth Trajectory by Reducing Auxin

Transporter PIN2 Protein Levels

in Arabidopsis thaliana.

Front. Plant Sci. 12:632676.

doi: $10.3389 / \mathrm{fp} / \mathrm{s} .2021 .632676$

\section{Abscisic Acid Regulates the Root Growth Trajectory by Reducing Auxin Transporter PIN2 Protein Levels in Arabidopsis thaliana}

\author{
Qijun Xie ${ }^{1,2 *}$, Jemaa Essemine ${ }^{3}$, Xiaochen Pang ${ }^{1}$, Haiying Chen ${ }^{1}$, Jing Jin ${ }^{1}$ and \\ Weiming Cai ${ }^{1 *}$
}

${ }^{1}$ Laboratory of Photosynthesis and Environment, CAS Centre for Excellence in Molecular Plant Sciences, Shanghai Institute of Plant Physiology and Ecology, Chinese Academy of Sciences, Shanghai, China, ${ }^{2}$ State Key Laboratory of Chemo/Biosensing and Chemometrics, College of Biology, Hunan Key Laboratory of Plant Functional Genomics and Developmental Regulation, Hunan University, Changsha, China, ${ }^{3}$ National Key Laboratory of Plant Molecular Genetics, CAS Centre for Excellence in Molecular Plant Sciences, Shanghai Institute of Plant Physiology and Ecology, Chinese Academy of Sciences, Shanghai, China

The root is in direct contact with soil. Modulation of root growth in response to alterations in soil conditions is pivotal for plant adaptation. Extensive research has been conducted concerning the adjustment of root elongation and architecture in response to environmental factors. However, little is known about the modulation of the root growth trajectory, as well as its hormonal mechanism. Here we report that abscisic acid (ABA) participated in controlling root growth trajectory. The roots upon ABA treatment or from ABA-accumulation double mutant cyp707a1,3 exhibit agravitropismlike growth pattern (wavy growth trajectory). The agravitropism-like phenotype is mainly ascribed to the compromised shootward transportation of auxin since we detected a reduced fluorescence intensity of auxin reporter DR5:VENUS in the root epidermis upon exogenous ABA application or in the endogenous ABA-accumulation double mutant cyp707a1,3. We then tried to decipher the mechanism by which ABA suppressed shootward auxin transport. The membrane abundance of PIN2, a facilitator of shootward auxin transport, was significantly reduced following ABA treatment and in cyp707a1,3. Finally, we revealed that ABA reduced the membrane PIN2 intensity through suppressing the PIN2 expression rather than accelerating PIN2 degradation. Ultimately, our results suggest a pivotal role for ABA in the root growth trajectory and the hormonal interactions orchestrating this process.

Keywords: abscisic acid, PIN-FORMED, shootward auxin transport, vertical growth index, root growth trajectory, agravitropism-like

Abbreviations: ABA, abscisic acid; ABF, ABRE BINDING FACTOR; BSA, Bovine Serum Albumin; ConcA, Concanamycin A; EZ, elongation zone; HPLC, high-performance liquid chromatography; MS, Murashige and Skoog; PBS, Phosphate buffer saline; PIN, PIN-FORMED; PI, propidium iodide; QC, quiescent centre; SAT, shootward auxin transport; VGI, vertical growth index. 


\section{INTRODUCTION}

Due to their sessile properties, the organ growth of plants exhibits plasticity. Since the root is located in a direct contact with the soil environment, its growth plasticity is indispensable for the plant adaptation. At least three parameters describe root growth: growth/elongation rate, architecture and growth trajectory/direction (e.g., grow straight or flexuous). Root growth concerning the 3 parameters varies frequently in the face of unstable soil environments. For instance, when roots encounter soil drought, the growth rate of the primary root will be significantly accelerated to search for water in deep soil (Xu et al., 2013). When the soil has a low nitrogen content, plants may reduce the numbers of lateral roots while increasing the length of each lateral root (Walch-Liu et al., 2006; Postma et al., 2014). Such adjustments would confer a larger, broader root system, which is favorable for nitrogen acquisition. Therefore, growth modulation represents a strategy for plants to survive in changeable circumstances, especially stressful conditions.

The regulation of the third growth parameter, growth trajectory represents another important strategy of plant adaptation. When gravity constitutes the sole environmental factor of concern, the root grows in a straight and centripetally downward manner into the soil. However, in nature, due to heterogeneous soil environments (such as hard solid) most root doesn't grow straitly (Lee et al., 2020). Apparently, modulation of root growth trajectory serves as a widely adopted and basic mechanism of plant adaption. Root growth trajectory has brought attentions from researchers and/or scientists (Cassab et al., 2013; Su et al., 2017; Lee et al., 2020). Considering that the existing terms used to describe root growth trajectory, such as waving and coiling, are mostly qualitative or semiquantitative (Mullen et al., 1998; Roy and Bassham, 2014). Grabov et al. (2005) established a set of parameters to estimate the wavy amplitude of root growth trajectory. Interestingly, they found that mutant lines (e.g., thr 1 and $\operatorname{axr} 2$ ) showing curved root also had alterations in the abilities of their roots to adapt to several environmental factors, including physical obstacles, light or gravity (Grabov et al., 2005; Sato et al., 2014). Therefore, flexibility of root growth trajectory/direction may boost (or strengthen) the plant ability to adapt to the changes in soil conditions.

Some types of growth trajectory show some typical characteristics. For example, the growth trajectory of a waving root exhibits a significantly sinusoidal growth pattern. However, less is known about the physiological relevance and the underlying molecular mechanisms of those curved growth trajectory without typical characteristics (unlike waving or coiling). Recently, it has been reported that the root growth trajectory could be regulated by several biotic/abiotic stresses, including high salinity, hydrogen peroxide, soil hypoxia and bacteria (Sun et al., 2008; Eysholdt-Derzso and Sauter, 2017; Zhou et al., 2018; Jimenez-Vazquez et al., 2020). For example, curvature of root growth trajectory was exacerbated with increasing the $\mathrm{NaCl}$ medium content (Sun et al., 2008). The authors further suggested that the curved phenotype might be associated with compromised auxin signaling (Sun et al., 2008). Moreover, hydrogen peroxide could also induce enhanced deviation to gravity vector by disrupting the membrane localization of the auxin efflux carriers PIN-FORMED 1 (PIN1) and PIN2 (Zhou et al., 2018). Therefore, curved root growth trajectory might uncover the coordination of the roots to adversities. Importantly, the phytohormone ABA plays an important role in the response to the abovementioned stress conditions (Neuman and Smit, 1991; Benech-Arnold et al., 2006; Veselov et al., 2008), and hydrogen peroxide has been suggested to be an intermediate of ABA signaling in the given phenotypes (Pei et al., 2000; Hung and Kao, 2004). This finding strongly implies that the stress-related hormone ABA serves as a mediator (in a concerted manner) between the stress conditions and the curved root growth trajectory/direction.

Here we report that the increased ABA level could elicit the wavy root growth trajectory. The phenotype elicited by the ABA resembles to that of roots agravitropism. However, the latter (gravitropism) appears not affected by ABA. We thus named or called this phenotype "agravitropism-like." We revealed that the agravitropism-like phenotype resulted from a reduction in the total shootward auxin concentration. Furthermore, ABA mitigates the shootward auxin content through suppressing PIN2 expression (an auxin efflux carrier), thus attenuating its membrane abundance. Such an attenuation of PIN2 abundance could also lead to agravitropism-like root. Overall, we present the regulatory effect of $\mathrm{ABA}$ on the root growth trajectory, and we further emphasized the role of ABA-auxin crosstalk in the context of root growth trajectory. These results may constitute a molecular network linking environmental factors to root growth trajectory.

\section{MATERIALS AND METHODS}

\section{Plant Material and Growth Conditions}

The following mutant lines were used in this study: a double mutant cyp707a1,3 generated by crossing cyp707a11 (At4g19230) with cyp707a3-1 (At5g45340) (Okamoto et al., 2006); The ABA signaling triple mutant, afb2abf3abf4 (Yoshida et al., 2015). A single mutant line in PIN2 (At5g57090), pin2-1 (CS8056) (Qi and Zheng, 2013); The auxin influx carrier mutant, aux1 (Marchant et al., 1999). the auxin receptor mutant line, tir11 (CS3798; Ruegger et al., 1998), purchased from the Arabidopsis Biological Resource Center (SALK). Ecotype Colombia (Col0 ) is the background of the five mutants: cyp707a1,3, pin2-1, afb2abf3abf4, aux1 and tir1-1.

The following fluorescent marker lines were used: PIN2:PIN2GFP (PIN2-GFP), GFP-tagged PIN2 protein in the Col-0 background (Birnbaum et al., 2003); DR5:VENUS, a synthetic auxin-responsive reporter line (Heisler et al., 2005); PIN1:PIN1GFP GFP-tagged PIN1 protein in the Col-0 background (Benková et al., 2003). PIN2:PIN2-EosFP (PIN2-EosFP). PIN2EosFP was generated following transformation process using the pCambia1300 backbone to insert PIN2:PIN2-EosFP in the plasmid (Dhonukshe et al., 2007).

PIN2-GFP/cyp707a1,3 was generated by crossing PIN2-GFP with cyp707a1,3. To obtain PIN2-EosFP, PIN2-EosFP/pin2-1 and PIN2-EosFP/cyp707a1,3, the pCambia1300-PIN2:PIN2-EosFP 
construct was introduced into the Col-0, pin2-1 and cyp7071,3 background, respectively. Among the $\mathrm{T}_{3}$ transgenic lines of PIN2-EosFP/pin2-1, one weak line and another strong one were chosen according to their PIN2 expression levels (low and high, respectively) and tagged as $\mathrm{W}$-rev-PIN2 and S-rev-PIN2. To express PIN2 specifically in the epidermis, PIN2-EosFP under the driver GL2 (At1g79840) promoter (from -2184 to +25 ) was introduced into the pin2-1 mutant. This epidermal PIN2 recovery line was named GL2:PIN2-EosFP in the current study.

Arabidopsis seeds were sterilized in $75 \%$ (V/V) ethanol for $5 \mathrm{~min}$ and sodium hypochloride for 15-30 $\mathrm{min}$. Then, they were washed 5-6 times with sterilized deionized water and sown onto $1 / 2 \mathrm{MS}$ medium (2.2 $\mathrm{g} \mathrm{l}^{-1} \mathrm{MS}$ salts, $1 \%$ sucrose, $10 \mathrm{~g}^{-1}$ bacto-agar $\mathrm{pH} 5.8$ with $1 \mathrm{M} \mathrm{KOH}$ ). Afterward, they were incubated for 2 -day at $4^{\circ} \mathrm{C}$ for stratification and dormancy removal, thereafter Petri dishes containing seeds were transferred to a growth chamber under controlled conditions (temperature, relative humidity, $\mathrm{RH}$ and photoperiod). These parameters were set as follows: $22^{\circ} \mathrm{C}, 80 \%(\mathrm{RH})$ and $16 / 8$-h light/dark.

\section{Measurement of VGI and Gravitropic Bending Rate}

To measure the root VGI following ABA treatment, 5-dayold seedlings geminated on $1 / 2 \mathrm{MS}$ were transferred onto $1 / 2$ MS medium either with or without ABA. Three days after transfer, the seedlings were photographed for subsequent VGI measurement. The root VGI was measured using ImageJ software according to the photos (version Java 1.8.0_112) ${ }^{1}$. For the measurements of root gravitropic bending rates, 5-day-old Col0 seedlings were transferred onto the corresponding media (with/without ABA addition) one day before gravistimulation. Roots were placed horizontally for gravistimulation. Photos were obtained at 3, 6, and $9 \mathrm{~h}$ after gravistimulation. Root gravitropic bending rates were assessed according to the images obtained at each time point $(3,6$, and $9 \mathrm{~h})$ using an Image J software.

For roots that have not undergo the transfer operation (Figures 2, 8 and Supplementary Figure 5), VGI is equal to the ratio of vertical root length to the total root length (Figure 1A), i.e., $L y / L$. When seedlings were transferred onto a new medium for hormonal treatments (ABA and/or IAA), VGI represented the ratio of increased root depth $(\Delta L y$; Figure 1B, blue doubleheaded arrow) to the elongated (supplemental) root length $(\Delta L$; Figure 1B, brown curve) after transfer, i.e., $\Delta L y / \Delta L$.

\section{Plant Hormonal Treatments}

For the VGI measurements under phytohormone (ABA and IAA) treatments, 5-day-old seedlings grown on 1/2 MS medium were transferred onto various new media containing the relevant plant hormones (ABA and/or IAA). Three d after transfer, the seedlings were photographed for subsequent VGI determination. When seedlings are prepared for confocal microscopy, the hormonal treatment endured only $1 \mathrm{~d}$, after which the samples were loaded for observation using confocal microscope.

\footnotetext{
${ }^{1}$ https://imagej.nih.gov/ij/
}

\section{Quantification of ABA Content}

ABA extraction from the root tip of the WT and the double mutant cyp707a1,3 was conducted following the method of Pan et al. (2010). Notably, seeds were mounted very tightly on the plate for easy sampling. Totally $50 \mathrm{mg}$ of root tip (fresh weight; $\sim 4 \mathrm{~mm}$ in length) collected from either WT or cyp707a1,3 was ground in liquid nitrogen. Subsequently, $20 \mathrm{ng}$ of $\left[{ }^{2} \mathrm{H}_{6}\right] \mathrm{ABA}$

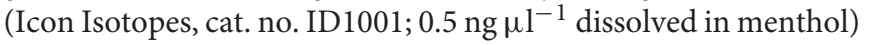
was added as the internal standard. Subsequently, $500 \mu \mathrm{l}$ extraction buffer was added to each sample. The extraction buffer contains 2-propanol $/ \mathrm{H}_{2} \mathrm{O} / \mathrm{HCl}(2: 1: 0.002 \mathrm{v} / \mathrm{v} / \mathrm{v})$. The samples were mixed well using a shaker operating at $100 \mathrm{rpm}$ under $4^{\circ} \mathrm{C}$ for $30 \mathrm{~min}$. Then, $1 \mathrm{ml}$ dichloromethane was added and the shaking was continued for an additional 30 min under the same conditions. Afterward, the samples were centrifuged at $13,000 \mathrm{rpm}$ at $4^{\circ} \mathrm{C}$ for $5 \mathrm{~min}$. Then, $\sim 900 \mu \mathrm{l}$ liquid of the lower phase was gently transferred and the upper phase discarded. A gentle handling is recommended to avoid disruption of the pellet (solid phase) located between the two liquid phases (lower and upper). Subsequently, the samples ( 900 $\mu \mathrm{l}$ ) were concentrated using nitrogen flow without complete drying. The samples were then dissolved in $0.1 \mathrm{ml}$ methanol and stored at $-80^{\circ} \mathrm{C}$ for a later HPLC analysis. The ABA content in each sample was determined on an Agilent liquid chromatography-tandem mass spectrometry device system equipped with a ZORBAX Eclipse XDB-C18 column as described previously (Yano et al., 2009; Weng et al., 2015).

\section{RNA Isolation and Quantitative Real-Time Polymerase Chain Reaction (qRT-PCR)}

Total RNA from the root tip of Col-0, single mutant (pin2-1), double mutant (cyp707a1,3) and transgenic lines (W-rev-PIN2 and S-rev-PIN2) was extracted following the manufacturer's instructions supplied with the total RNA isolation kit (RNeasy, QIAGENE, No. 74904). One hundred nanograms of the isolated RNA from each sample was used to reverse transcribe (synthesis) the first-strand cDNA using ReverTra Ace qPCR RT MASTER MIX (with gDNA remover; TOYOBO, No. FSQ-301). The qRT-PCR was conducted using Real-time PCR Master Mix (TOYOBO, No. QPK-101) with 3 biological replicates for each sample. The parameters used for qRT-PCR cycling were as follows: $95^{\circ} \mathrm{C}$ for $10 \mathrm{~s}, 55^{\circ} \mathrm{C}$ for $20 \mathrm{~s}, 72^{\circ} \mathrm{C}$ for $20 \mathrm{~s}$. The primer sequences, GCCGAGAGCTTCTAGCTTTAA (forward) and CGCCGTAGCTATTAGTGTAACC (reverse), were used for the amplification of PIN2. The primer sequences, TCGGTCGTTGTATTGTGCTTT (forward) and CCAGATGCTCATTACACACTCA (reverse), were used for the amplification of $R A B 18$ (At5g66640). For the reference gene Actin2, GCACCCTGTTCTTCTTACCG (forward) and AACCCTCGTAGATTGGCACA (reverse) were used. The primer sequences AACTTTGGTGGTTTGTGTTTTGG (forward) and TCGACTTGTCATTAGAAAGAAAGAGATAA (reverse) were used for the amplification of another reference, $U B Q 10$. Because the results using Actin2 or UBQ10 as the reference were identique, we used Actin2 to normalize PIN2 
A

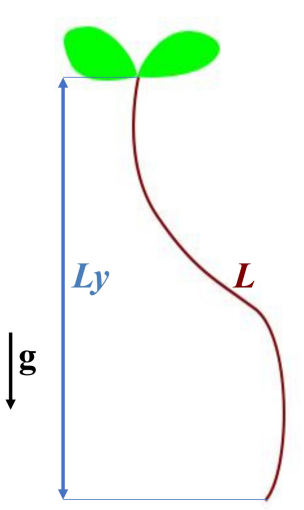

Vertical growth index (VGI)

VGI $=L y / L$

B

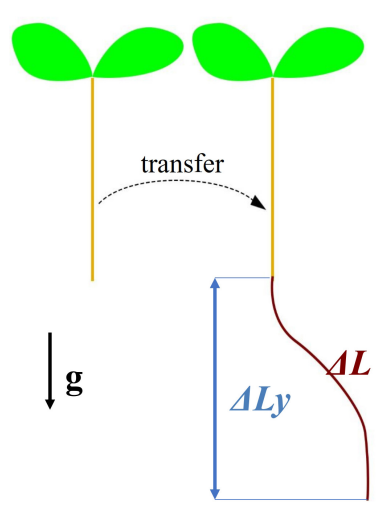

Vertical growth index (VGI)

VGI $=\Delta L y / \Delta L$
C

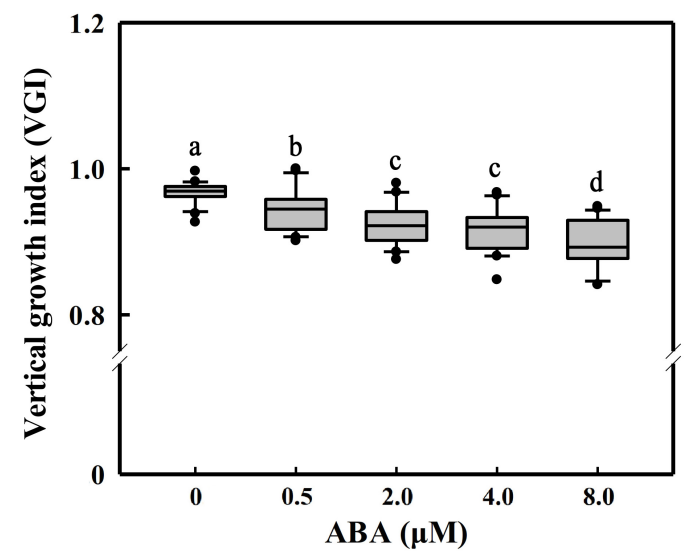

D Ctrl Ctrl $\quad 0.5 \overline{\mu M}$ $0.5 \mu \mathrm{M} \quad 2.0 \mu \mathrm{M}$ ABA
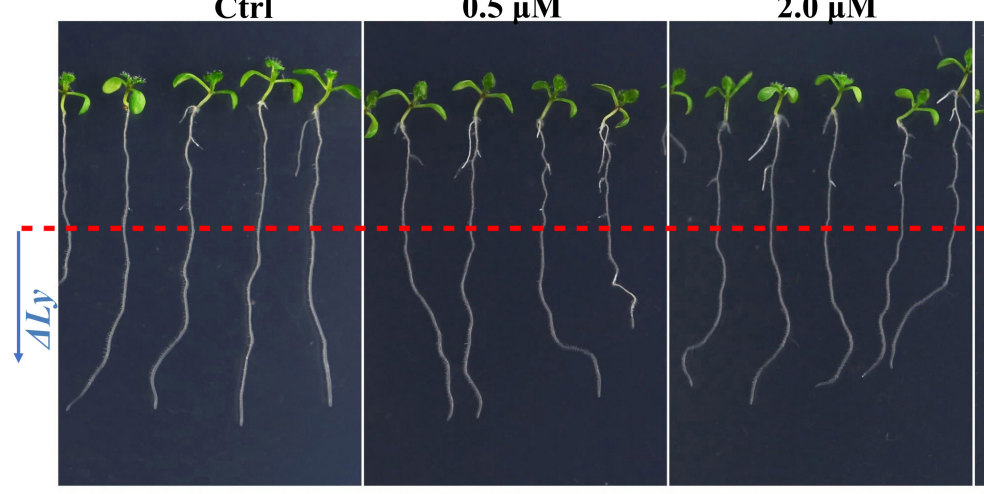
$4.0 \mu \mathrm{M}$ $8.0 \mu M$

FIGURE 1 | Definitions of the VGl and root VGI in the presence of different ABA concentrations. (A) A schematic diagram defining VGl before the transfer operation. $L y$ : vertical primary root length (root depth; the blue double-headed arrow); $L$ : total root length (the brown curved line). VGl equals the ratio of $L y$ to $L$ : VGl $=L y / L$. (B) A simple schematic diagram illustrating the VGl of seedlings that have undergone the transfer operation. Seedlings grown on 1/2 MS medium, then transferred on a new medium (with ABA). $\Delta L y$ and $\Delta L$ of the root were measured 3-day after transfer. $\Delta L y$ : increased root depth after transfer (blue double-headed arrow); $\Delta L$ : increased root length after transfer (brown curve). VGI denotes the ratio of $\Delta L y$ to $\Delta L: V G I=\Delta L y / \Delta L$. (C) Box plot showing the root VGl in the presence of different $\operatorname{ABA}$ concentrations $(0.5,2,4$, and $8 \mu \mathrm{M})$. Five-day old seedlings before being grown on $1 / 2 \mathrm{MS}$ medium were transferred onto $1 / 2 \mathrm{MS}$ media containing different ABA concentrations. As the control (Ctrl), seedlings were transferred onto the common 1/2 MS medium without any additions. At 3-day after transfer, the seedlings were photographed and then VGI measured. $n>30$. (D) Representative images of Col-0 seedlings in the absence (Ctrl) or presence of different ABA concentrations as mentioned in panel (C). The bottom root parts blow the red-dashed line represent the elongated roots after transfer.

expression. The relative PIN2 expression compared with Actin2 was calculated using the $2^{-\Delta \Delta C T}$ method as described previously (Livak and Schmittgen, 2001).

\section{Auxin Immunofluorescence}

Root tips obtained from 5-day-old Col-0 and cyp707a1,3 seedlings were prefixed at $4^{\circ} \mathrm{C}$ for $30 \mathrm{~min}$ in cross-linking buffer that could cross-link the carboxyl group of free IAA to structural proteins and preserve its antigenicity (Márquez-López et al., 2018). This cross-linking buffer consists of $3 \% \mathrm{~N}$-(3dimethylaminopropyl)- $N^{\prime}$-ethylcarbodiimide hydrochloride (EDAC; Sigma, No. 03449) in $1 \times$ phosphate-buffered saline $(1 \times$ PBS). After prefixation, the samples were fixed in FAA solution (formaldehyde alcohol acetic acid; $50 \%$ ethanol, 5\% acetic acid, $37 \%$ formaldehyde; $18: 1: 1)$ overnight at $4^{\circ} \mathrm{C}$. The samples were then washed 3 times with $1 \times$ PBS for 5 min each.
Thereafter, the cell walls were digested with $1 \%$ Macerozyme R-10 and $1 \%$ Driselase (dissolved in $1 \times$ PBS) for $18 \mathrm{~min}$ at $37^{\circ} \mathrm{C}$. Subsequent membrane permeabilization was conducted after digestion by submerging the samples in the permeabilization solution (3\% IGEPAL CA-630 and 10\% DMSO in $1 \times$ PBS) for $20 \mathrm{~min}$ at $37^{\circ} \mathrm{C}$. The samples were then washed 5 times with $1 \times$ PBS for $5 \mathrm{~min}$ each. Afterward, the samples were submerged in blocking buffer (3\% BSA dissolved in $1 \times$ PBS) for $90 \mathrm{~min}$ at room temperature $\left(20-22^{\circ} \mathrm{C}\right)$. Thereafter, they (samples) were incubated overnight with mouse anti-IAA monoclonal antibody (1:200 dissolved in 3\% BSA in PBS); Sigma) at $4^{\circ} \mathrm{C}$ and subsequently washed 3 times with $1 \times$ PBS. Finally, the samples were incubated with Alexa Fluor 488 goat anti-mouse IgG secondary antibody (1:100; Abcam) for $2 \mathrm{~h}$ at $37^{\circ} \mathrm{C}$ followed by 3 washes $(5 \mathrm{~min}$ each) with $1 \times$ PBS. For examination, the root tips were mounted onto glass slides for confocal microscopy 

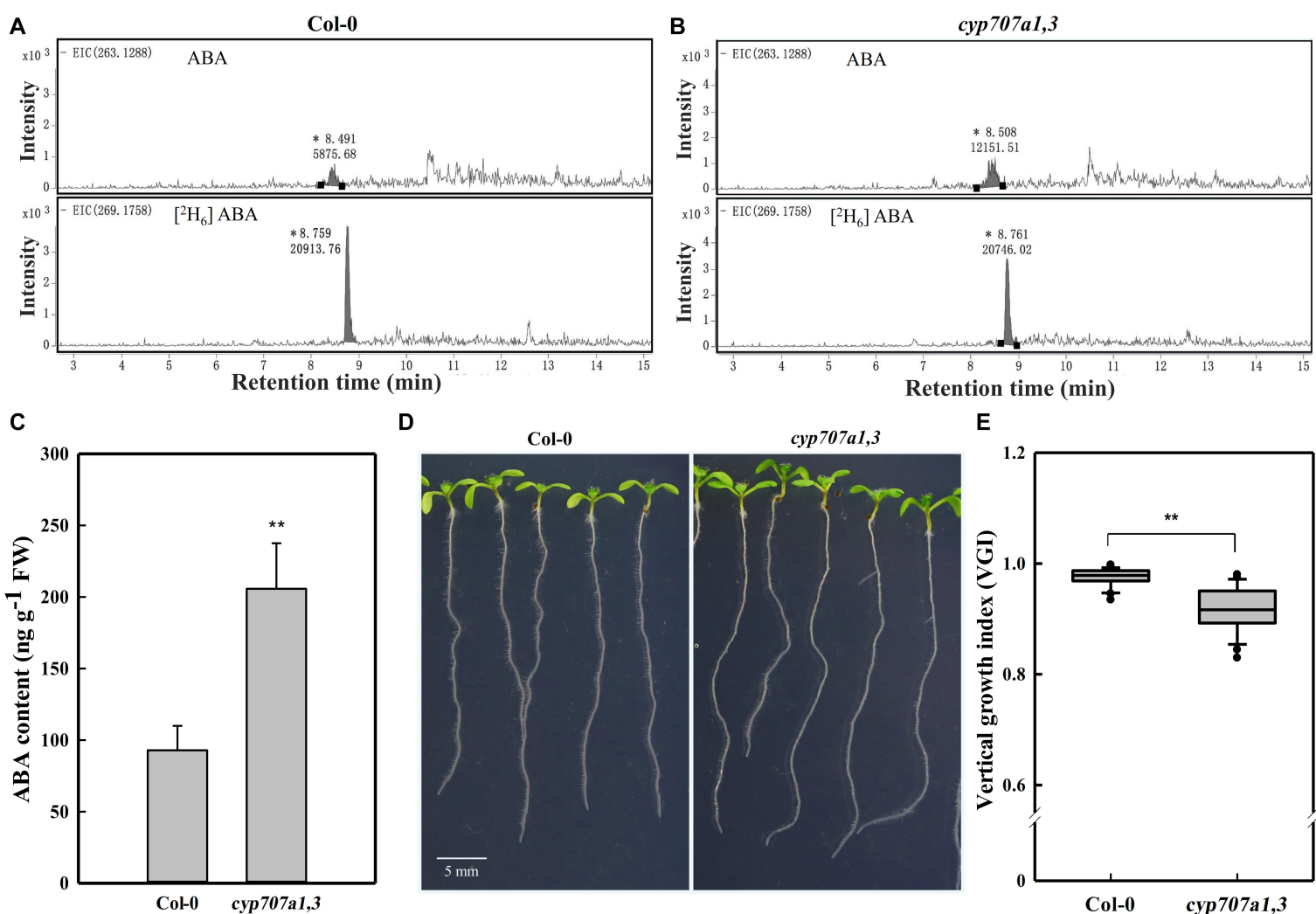

FIGURE 2 I HPLC-MS dependent quantification of ABA contents in primary roots, the root VGl of WT (Col-0) and cyp707a1,3. (A) Extracted ion chromatograms (ElCs) of ABA and its internal standard $\left[{ }^{2} \mathrm{H}_{6}\right]$ ABA from extracts of 7 -day-old Col-0 root tip. ElCs of ABA (upper panel) and [ $\left.{ }^{2} \mathrm{H}_{6}\right]$ ABA (bottom panel) were extracted from masses of $263.1288 \pm 10$ and $269.1758 \pm 10$ for $\mathrm{ABA}$ and $\left[{ }^{2} \mathrm{H}_{6}\right] \mathrm{ABA}$, respectively. Integral peak of $\mathrm{ABA}$ (retention time: $\left.\sim 8.49 \mathrm{~min}\right)$ and $\left[{ }^{2} \mathrm{H}_{6}\right] \mathrm{ABA}($ retention time: $\sim 8.75 \mathrm{~min}$ ) were measured for the subsequent evaluation of ABA content. (B) EICs of ABA and the internal standard $\left[{ }^{2} \mathrm{H}_{6}\right]$ ABA from the cyp707a1,3 double mutant extract. The integral peaks of $\mathrm{ABA}$ (retention time: $\sim 8.50 \mathrm{~min}$ ) and $\left[{ }^{2} \mathrm{H}_{6}\right] \mathrm{ABA}$ (retention time: $\sim 8.76 \mathrm{~min}$ ) were measured to qualify the ABA content in the root tip of cyp707a1,3. (C) The ABA content in the primary root tip of Col-0 and cyp707a1,3. ABA contents were evaluated according to the ratio of the integral peak of $\mathrm{ABA}$ and its respective standard $\left[{ }^{2} \mathrm{H}_{6}\right]$ ABA. Each bar represents the mean of 3 biological samples and 3 technical replications \pm SE. (D) Root VGl of Col-0 and cyp707a1,3. The images display 7-day-old seedlings grown on 1/2 MS medium. (E) The box plot presents the root VGl of the WT (Col-0) and double mutant cyp707a1,3. $n>30 .{ }^{*}$ and ${ }^{* *}$ represent $p<0.05$ and $p<0.01$ respectively.

observations. The immunofluorescence protocol was performed as documented previously with slight modifications (Pasternak et al., 2015; Márquez-López et al., 2018).

\section{Confocal Microscopy and Fluorescence Intensity Measurements}

Root tips were mounted on glass slide for confocal microscopy. In this study, an Olympus FV $1000^{2}$ was used for confocal microscopy. Unless otherwise specified, the parameters for confocal imaging between different trails or time points within an independent experiment were completely the same. For DR5:VENUS (YFP), we used the 515-nm band of an argon laser for excitation. A 488-nm wavelength was used for GFP; $559 \mathrm{~nm}$ was used for propidium iodide (PI) excitation. The 2 image types for the DR5:VENUS signal in Figure 3 were obtained by scanning through different sections of roots. The cross-section view was generated by scanning through the middle of a root (Figure 4A,

\footnotetext{
${ }^{2}$ https://www.olympus-lifescience.com.cn/
}

middle panel). The top view was generated by scanning through the top surface of the root tip (Figure $\mathbf{4 A}$, right panel). The photoconversion operation shown in Figure 9 was performed by exposing the whole root tip to $405-\mathrm{nm}$ wavelength radiation for $30 \mathrm{~s}$. Hence, 5-day-old seedlings were used in these experiments.

The raw data for fluorescent images generated using an Olympus FV1000 confocal microscope were manipulated using Image-Pro Plus software (v6.0), in which membranes were outlined automatically with the same threshold gray value. Average gray values were used to represent the fluorescence intensities, which were finally normalized to their respective control (explained in detail in the figure legend).

\section{Statistical Data Analysis}

The data were collected in Microsoft Excel 2019. We performed ANOVA followed by Tukey's pairwise comparisons at a $P$-value $<0.05$ for the following analyses: ABA content in Col- 0 and cyp707a1,3 (Figure 2C); root VGI of Col-0 and cyp707a1,3 (Figure 2E); RAB18 expression in Col-0 and abf2abf3abf4 

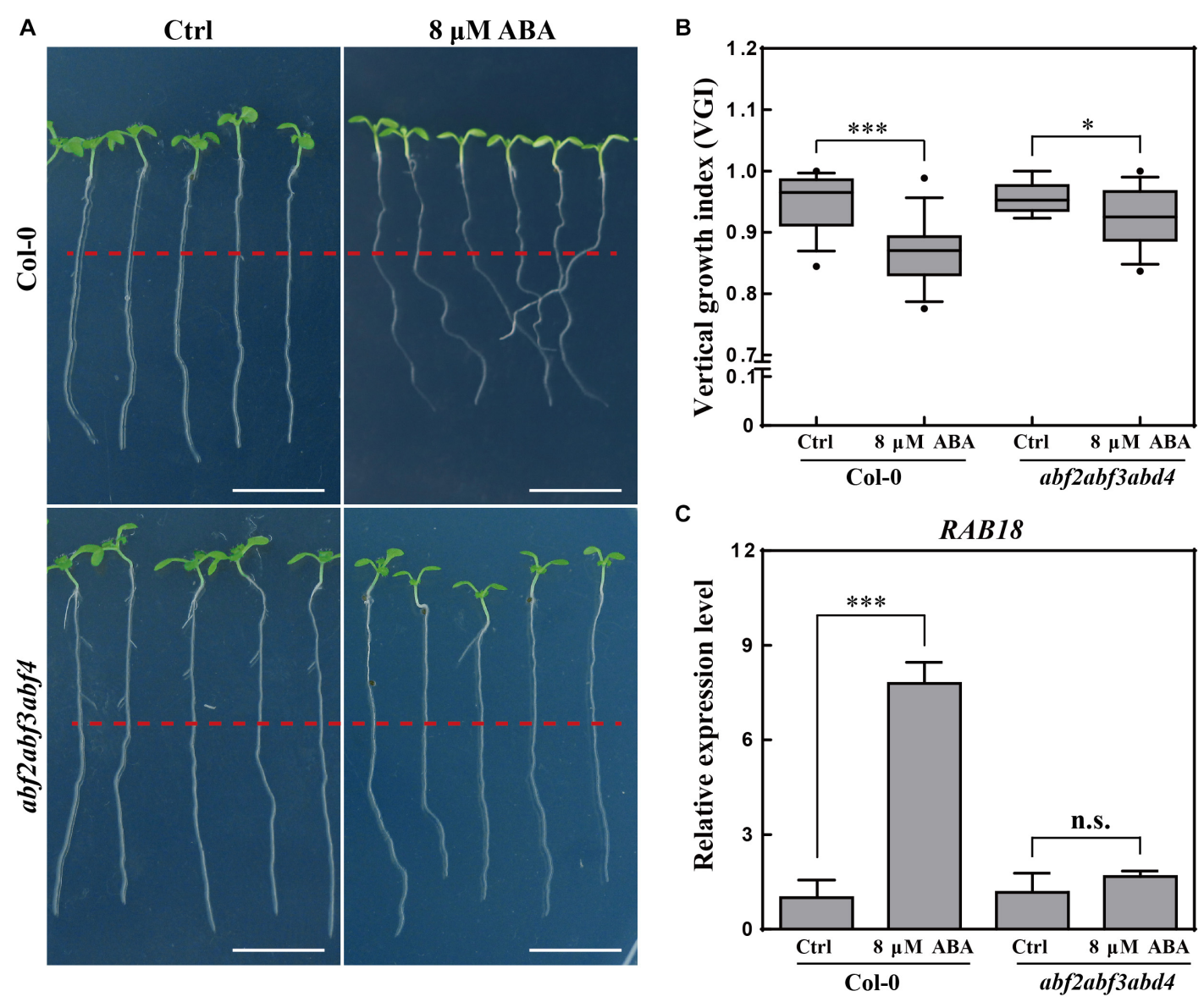

FIGURE 3 | Root VGI of Col-0 and abf2abf3abf4 in response to ABA treatment. (A) Representative images of Col-0 and abf2abf3abf4 seedlings for control and following ABA treatment. 5-day-old seedlings were treated with $8 \mu \mathrm{M} \mathrm{ABA}$; the photographs were taken 3-day after transfer. White bar represents a scaling bar of $1 \mathrm{~cm} . n>30$. (B) Root VGI of Col-0 and abf2abf3abf4 seedlings for control and following ABA treatment. Each bar represents the mean of 30 independent measurements $\pm \mathrm{SE}$. (C) Relative expression level of RAB18 in roots of Col-0 and abf2abf3abf4 with and without ABA treatment. Each bar represents the mean of 3 biological samples and 3 technical replicates \pm SE. * and ${ }^{* * *}$ represent $p<0.05$ and $p<0.001$ respectively.

(Figure 3B); VGI of Col-0 and abf2abf3abf4 in control or following ABA treatment (Figure 3C). IAA/Alexa 488 intensity in Col-0 and cyp707a1,3 (Figure 4F); IAA/Alexa 488 signal coverage in the epidermis of Col-0 and cyp707a1,3 (Figure 4I). The statistical analysis for the other parameters, we performed ANOVA followed by Tukey's multiple comparison tests at a $P$-value $<0.05$.

Concerning the box plots, the central vertical bar in the box represents the median, and the box represents the interquartile range. Two plot whiskers span 1.5 times the interquartile range of the distribution. The dots outside of whiskers denote outliers.

\section{RESULTS}

\section{ABA Elicits an Agravitropism-Like Root Phenotype}

To investigate whether ABA could control the root growth trajectory, we first transferred 5-day-old WT (Col-0) seedlings onto $1 / 2$ Murashige and Skoog (MS) medium containing different concentrations of ABA (Figure 1). We implemented a transfer operation due to the inhibitory effect of $\mathrm{ABA}$ on germination (Ackerson, 1984; Finkelstein et al., 2002; Liu et al., 2013). We found that ABA could elicit an agravitropism-like phenotype, which presents wavy growth trajectory/direction (Figure 1). To quantify the wavy amplitude of root growth trajectory, we adopted a reported parameter, the vertical root growth index (VGI; Figure 1A; Grabov et al., 2005). To avoid the inaccuracy that can be introduced by the transfer operation, we provided an additional definition to VGI for the transferred seedlings. Thus, VGI of the transferred seedling equals the ratio of increased root depth $(\Delta L y$; the blue double-headed arrow in Figure 1B) to the elongated root length $(\Delta L y$; the curved line in Figure 1B) after transfer, i.e., $\Delta L y / \Delta L$ (Figure 1B). Briefly, a lower VGI represents an exaggerated curvature or wavy amplitude of root growth trajectory. Hence, we transferred 5day-old seedlings onto mediums containing 0.5-8 $\mu \mathrm{M}$ ABA. Notably, the used ABA concentrations that cover relatively a broader range, which may reflect various environmental conditions. Using various ABA concentrations, we found that 
A

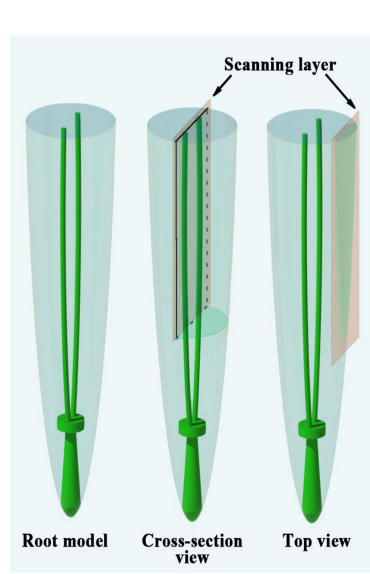

E

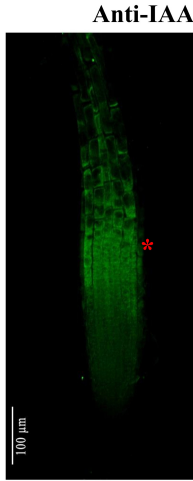

Col-0
B

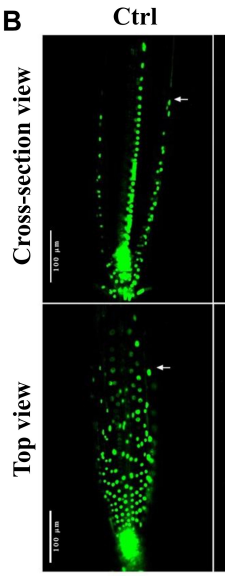

F

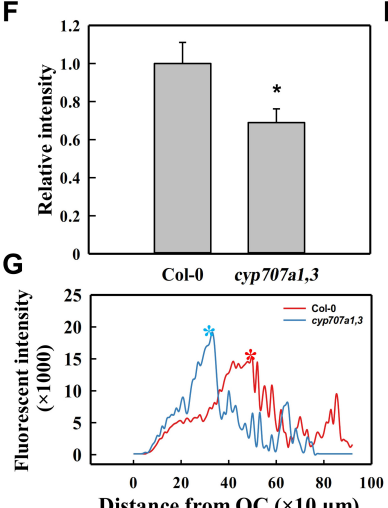

ABA

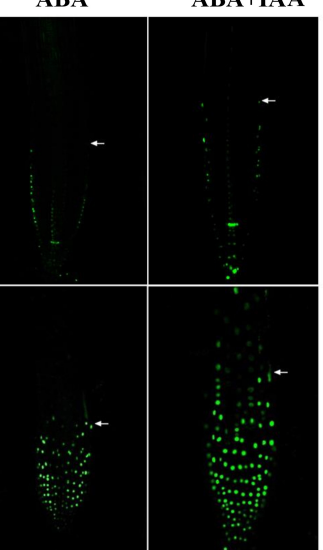

H

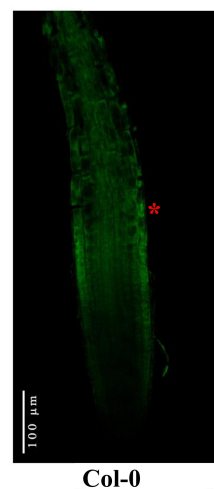

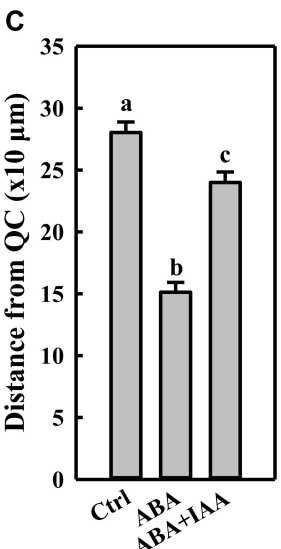

D

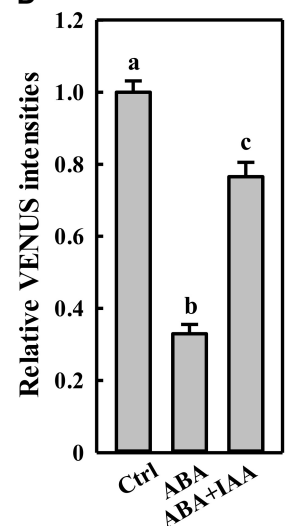

I

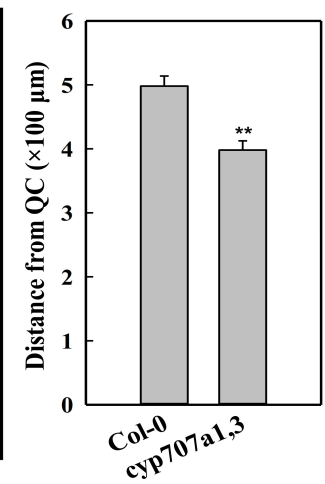

FIGURE 4 | DR5:VENUS signal in the root tip following different treatments, and immunofluorescence of IAA in the root tip of Col-0 and cyp707a1,3. (A) Schematic diagram of the scanning layers of the cross-section (middle panel) and top views (right panel). The cross-section view refers to the image generated by scanning through the middle of the root tip (middle panel). The top view was generated by scanning through the surface of a root. (B) Cross-section and top views of DR5:VENUS signal in the control root tip and those treated with plant hormones. Five-day-old DR5:VENUS seedling were transferred onto control (1/2 MS without hormonal addition), ABA (1/2 MS with $8 \mu \mathrm{M} \mathrm{ABA})$ or ABA + IAA (1/2 MS with $8 \mu \mathrm{M}$ ABA together with $5 \mathrm{nM} I \mathrm{AA})$ media. Following a single day of treatment, the seedlings were loaded onto a confocal microscope for imaging. White arrowheads indicate the ends of DR5:VENUS signals. White triangles indicate PIN2-GFP signals in cells of EZ. (C) Coverage of SAT as reflected by the distance from the end of the epidermal DR5:VENUS signal (panel b, white arrowheads) to the QC. Measurements were conducted according to the cross-section view in the panel (B). (D) Relative epidermal DR5:VENUS intensities following the same treatments as in panel (B). Fluorescence intensities were normalized to the control. Measurements were conducted according to the top view in panel (B). (E) Top view of the anti-IAA/lexa Fluor 488 (IAA/Alexa 488) signal in Col-0 and cyp707a1,3. Primary roots (PRs) of 5-day-old Col-0 and cyp707a1,3 seedlings were immunodetected using anti-IAA/Alexa Fluor 488. Fluorescence intensities were normalized to Col-0. (F) Total intensity of IAA/Alexa 488 in the root tip of Col-0 and cyp707a1,3.

(G) Vertical profiles of IAA/Alexa 488 signal in the epidermis. Line profiles were generated from the epidermal IAA/Alexa 488 signal as shown in panel (E), according to its fluorescence intensity along the vertical direction of the root. (H) Cross-section view of IAA/Alexa 488 in the root tip. The stars (red and blue) in this panel, as well as those in panels (E,G), indicate the intensity peak of the IAA/Alexa 488 signal in Col-0 (red) and cyp707a1,3 (blue). $n=15$. (I) Coverage of acropetal auxin flux (AAF) evaluated by the distance from the intensity peak of the epidermal IAA/Alexa 488 signal [panel (H), red and blue stars] to QC. Measurements were performed according to the cross-section view. For the histograms shown in panels (C,D), each bar data represents the mean of 30 independent measurements \pm SE. * and ${ }^{\star \star}$ represent $p<0.05$ and $p<0.01$ respectively.

ABA significantly reduces the root VGI (Figures 1C,D), indicating that the root growth trajectory could somehow be regulated by $\mathrm{ABA}$.

The addition of ABA may affect the physicochemical properties of the medium, and thus the agravitropism-like phenotype could result from the altered surface characteristics of the medium. To exclude this possibility, we focused to determine whether the agravitropism-like phenotype could be manifested by an endogenous ABA accumulation. Herein, we investigated the VGI in the double ABA-degradation mutant, cyp707a1cyp707a3 (cyp707a1,3 hereafter), which is known by its higher ABA content in the dry seeds by approximately 20 folds if compared to WT (Okamoto et al., 2006). Since both CYP707A1 and CYP707A3 are mainly expressing in the roots (Winter et al., 2007) and our current work focuses on root growth, we accordingly expect an increase in the endogenous $\mathrm{ABA}$ content in the primary root of the cyp707a1,3 double mutant. To confirm this finding, we quantified the ABA content in the primary root of cyp707a1,3 using high-performance liquid chromatography (Figures 2A,B), and verified corresponding intensity peak via the tandem mass spectrometry (Supplementary Figure 1). We detected an approximately 2 folds higher $\mathrm{ABA}$ content in the primary root tip 
of cyp707a1,3 compared to the WT (Figures 2A,C). In line with the results obtained for exogenous ABA application, the VGI of cyp707a1,3 was significantly lower than that in the WT $(p<0.01$; Figures 2D,E). Therefore, instead of the alteration of surface characteristics of the medium, ABA accumulation represents the main reason for the agravitropism-like phenotype (Figures 1, 2).

To better understand the relationship between ABA and the agravitropism-like phenotype, a triple mutant line in three transcription factors of ABA signaling, the abf $2 a b f 3 a b f 4$ (Yoshida et al., 2015) was tested for its response to ABA treatments. The VGI of abf2abf3abf4 was far less sensitive upon ABA application (Figures $\mathbf{3 A}, \mathbf{B}$ ). To assess the ABA signaling in $a b f 2 a b f 3 a b f 4$, the expressions of a widely used ABA responsive gene $R A B 18$ (Yoshida et al., 2015) were quantified in WT and the mutant. Hence, the exogenous ABA $(8 \mu \mathrm{M})$ application considerably increases the RAB18 expression in WT, meanwhile its (RAB18) expression level in the abf2abf $3 a b f 4$ is found to be less sensitive to ABA treatment (Figure 3C). Therefore, the ABA-elicited agravitropism-like phenotype is impaired in the ABA signaling mutant. Otherwise, ABA affects the root growth trajectory through partially the ABRE BINDING FACTORs (ABFs) pathway.

\section{ABA Attenuates Shootward Auxin Content in the Root Tip}

Auxin is transported from the root cap back toward the root elongation zone (EZ) through an auxin efflux carrier PIN2 (Friml, 2003; Kramer and Bennett, 2006). The auxin transport in this direction was earlier termed shootward auxin transport (SAT; Friml, 2003; Kramer and Bennett, 2006). Certain SAT mutants (e.g., pin2) show a curved root growth trajectory and gravitropic growth pattern (Bennett et al., 1996; Chen et al., 1998; Müller et al., 1998; Marchant et al., 1999). Thus, the agravitropismlike phenotype induced by ABA likely also to be resulted from the alterations in SAT. Thus, we used the auxin reporter line DR5:VENUS to determine whether SAT could be influenced by ABA. Based on the cross-section view, which presents the clear signal ends in the epidermis (Figures 4A,B), we could observe a significantly reduced coverage of the DR5:VENUS signal in the epidermis upon ABA treatment (Figures 4B,C). Moreover, according to the top view, which provides an accurate information regarding fluorescence intensity (Figures 4A,B), the epidermal DR5:VENUS intensity was strongly reduced following ABA application (Figures 4B,D). Hence, the Reduction in both the signal intensity and coverage implies the mitigation in the shootward auxin content. It's worthy to note that auxin is not accumulated obviously in the root caps or QC although the impairment of its SAT. This results from a decreased rootward auxin transport as indicated by the reduced PIN1 abundance (Supplementary Figure 2), which is involved in the auxin transport from the up-ground source to QC and root caps.

To verify whether the endogenous ABA accumulation could cause the same attenuation in auxin content, we investigated the auxin distribution in the root tip of cyp707a1,3 and WT (Col-0) via immunofluorescence (Figures $4 \mathbf{E}, \mathbf{H}$ ). Using antiIAA/Alexa Fluor 488 to detect the auxin in the root tip, we thus demonstrated that the total anti-IAA/Alexa Fluor 488 (IAA/Alexa 488) intensity in the epidermis of cyp707a1,3 was significantly reduced compared to the WT (Figures $4 \mathrm{E}, \mathbf{F}$ ). As reflected by the vertical profile (signal intensity distribution along the vertical direction) of the top-view signal (Figure 4G), the IAA/Alexa 488 in cyp707a1,3 appears to reach its intensity peak much earlier (closer to quiescent centre; QC) than WT (Figures 4E,G, red and blue stars). In addition, the immunofluorescence signals in the $\mathrm{EZ}$ of the cyp707a1,3 double mutant were almost undetectable; however, the signal in the same region of WT was significantly stronger (Figures $4 \mathrm{E}, \mathrm{G}$ ). As shown by both the cross-section and top views (Figures $4 \mathbf{E}-\mathbf{H}$; indicated by red and blue stars), the distance from the QC to the intensity peak of IAA/Alexa 488 was shorter in cyp707a1,3 than in WT (Figure 4I). This indicates that the total auxin content of SAT was weakened in cyp707a1,3, since both the signal coverage and intensity were decreased (Figures 4E-I). Thus, both the exogenous and endogenous enhancements in ABA level could reduce the shootward auxin content.

The physical distance between QC and the end of DR5:VENUS signal could be affected if the ABA application causes a shorter meristem (Supplementary Figure 2; indicated by white arrowheads). In this regard, we employed a PI staining to visualize auxin distribution pattern in different root zones. In WT roots, auxin considerably distributed from the meristem zone to the EZ (including transition zone; Figure 5). Following ABA application, DR5:VENUS signals were mainly restricted to meristem (Figure 5). Hence, it appears that ABA triggering a reduction in DR5:VENUS coverage is not only attributable to the reduced meristem size, but also to the impaired SAT. Since roots bend after transfer onto an ABA medium, we decide to examine the epidermal cell elongation in the bending site of roots treated with ABA. The cells in convex and concave root face do not

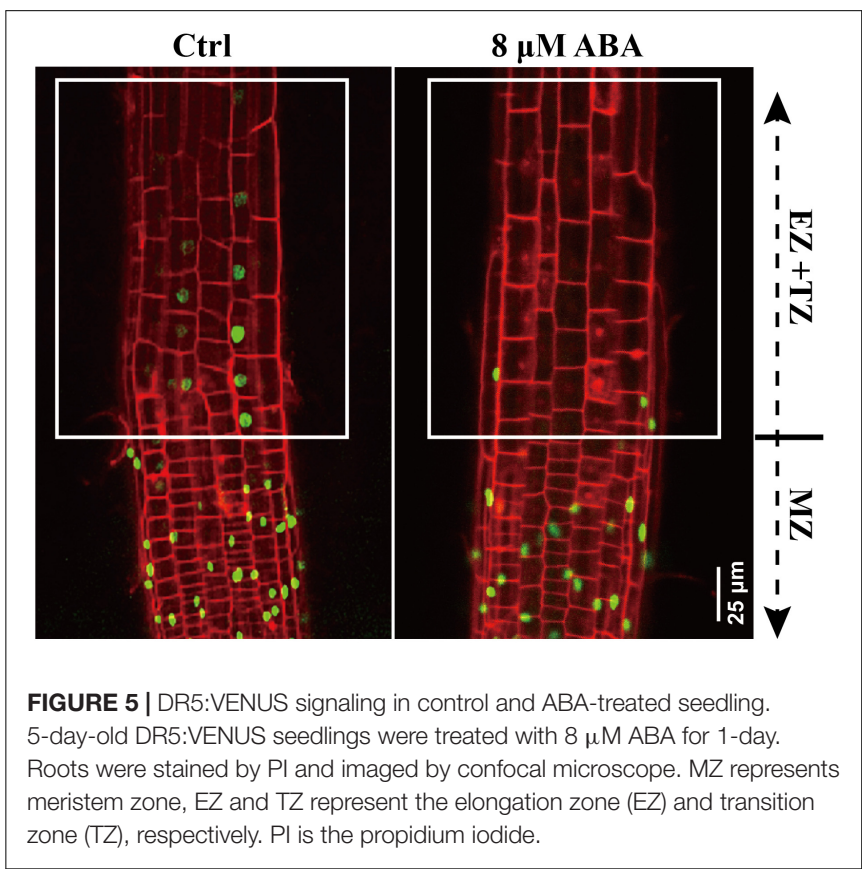


elongate equally (Supplementary Figure 3). Hence, we speculate that certain auxin level is required to maintain the symmetric cell growth in these regions. In conclusion, ABA impaired SAT confers low auxin concentration in EZ. Deficiency in auxin may not insufficient to maintain the symmetrical growth of cells in this region, further results in the root bending.

Since we demonstrated that ABA could attenuate the shootward auxin content (Figures 4, 5) and considering the assumption that SAT correlates with the root growth trajectory (Sun et al., 2008), we speculate that the agravitropism-like phenotype caused by ABA was due to the mitigated auxin level of SAT. To confirm this hypothesis, we added $50 \mathrm{nM}$ IAA together with $8 \mu \mathrm{M} \mathrm{ABA}$ to the medium. Compared to the ABA application alone, the DR5:VENUS intensity was increased in the epidermis following IAA application together with $\mathrm{ABA}$ (Figures 4B-D). This result suggests that the external application of IAA could partially compensate for the ABA-induced auxin diminution of SAT. Hence, we added IAA together with ABA to confirm whether the agravitropism-like phenotype could be rescued. As predicted, IAA greatly recovered the root VGI to the same level of the control (Supplementary Figure 4). However, in the auxin receptor mutant tir1-1, the compensatory effect was completely lost (Supplementary Figure 4). Accordingly, ABA-reduced root VGI could be rescued by the recovery of the shootward auxin level. Thus, ABA-attenuated the shootward auxin content could influence the root growth trajectory.

\section{PIN2 Is Required for the ABA-Modulated Root Growth Trajectory}

We mentioned in the precedent paragraph that ABA causes the agravitropism-like phenotype by reducing the auxin content of SAT. It is well documented that SAT is propelled (or driven) by an auxin efflux carrier, PIN2 (Müller et al., 1998; Shin et al., 2005). Hence, ABA-induced attenuation of the SAT very likely resulted from the reduced PIN2 abundance. To verify that, we investigated the PIN2 abundance and distribution in the root tip following ABA treatment. Hence, using a reporter line, PIN2:PIN2-GFP (PIN2-GFP, Thereafter), which expresses GFP-tagged PIN2 in WT (Col-0 background), we observed a significantly reduced PIN2 abundance upon $8 \mu \mathrm{M}$ ABA treatment (Figures 6A,B). We also crossed PIN2-GFP with cyp707a1,3 (double mutant) to introduce PIN2-GFP into cyp707a1,3, generating thus the PIN2-GFP/cyp707a1,3 line. In line with the results showing the $\mathrm{ABA}$ attenuation of the auxin content of SAT (Figure 4), the total membrane PIN2 intensity in PIN2-GFP/cyp707a1,3 was also weaker than in the WT (especially in EZ; Figures $\mathbf{6 A}, \mathbf{B}$, white triangles). Therefore, the accumulated $\mathrm{ABA}$ could reduce the membrane PIN2 abundance. The PIN2 is located in the epidermal membrane situated in the outermost layer of the root (Figure 6A; left panel, indicated by letter e) and the cortex layer directly adjacent to the epidermis (Figure 6A; left panel, depicted by the latter c). In this regard, we found that the decrease in PIN2-GFP intensity of cyp707a1,3 was mainly attributed to its attenuation in the epidermis $(\sim 40 \%$ reduction compared with Col- 0$)$ rather than the cortex $(\sim 15 \%$ reduction; Figure 6C).
We also analyzed the PIN2 expression level in response to ABA application or in cyp707a1,3 mutant. Thus, the PIN2 expression level upon either ABA application or in cyp707a1,3 was deceased compared to the WT (Figure 6D). Hence, the reduced PIN2 abundance is partially due to the suppression of PIN2 transcription. Besides, the PIN2 expression level between ABA application and cyp707a1,3 didn't show any significant difference; however, the PIN2 intensity in cyp707a1,3 is stronger than for the $\mathrm{ABA}$ treatment. Although both $\mathrm{ABA}$ application and mutant line generate higher $\mathrm{ABA}$ concentration, the $\mathrm{ABA}$ content in cyp707a1,3 appears more accurately controlled, because of the spatial and temporal pattern of CYP707A1 and CYP707A3. For example, the subcellular zonation of these two enzymes (CYP707A1 and CYP707A3) might result in the missing of $\mathrm{ABA}$ induced post-transcriptional regulation of PIN2 in cyp707a1,3. In the scenario of ABA application, the ABA level increases ubiquitously and therefore inhibits the PIN2 translation at the post-transcriptional level. Altogether, although PIN2 expression level in ABA-treated root and cyp707a1,3 seems equal (Figure 6D), the differences in post-transcriptional level may confer differences in PIN2 abundance.

Although we showed above that the attenuation in shootward auxin content could lead to the agravitropism-like root, it is possible that the reduction in membrane PIN2 abundance could also trigger the same phenotype. Moreover, it remains unclear whether $\mathrm{ABA}$ modulated the root growth trajectory through regulating the PIN2 abundance or via another unknown process. To decipher these possibilities, we recovered PIN2 in pin2-1 by introducing PIN2-EosFP into the mutant, and we obtained one strong line and another weak recovery line according to the PIN2 relative expression level. The EosFPtag conjugated to the PIN2 protein is a fluorescent protein (property of EosFP will be discussed latter; Lippincott-Schwartz and Patterson, 2008; Bourgeois et al., 2012). The weak allele (W-rev-PIN2) possesses a much higher expression level than pin2-1. However, its PIN2 expression level represents only 20\% of that in WT (Supplementary Figure 5A). Correspondingly, its VGI was higher than in pin2-1 but lower than that in WT (Supplementary Figures 5B,C). The strong recovery line (S-rev-PIN2) shows a similar expression level to WT and close VGI values. Therefore, the agravitropism-like phenotype positively correlates with the PIN2 expression level, signifying the involvement of PIN2 in controlling the root growth trajectory. We then assessed the VGI of the pin2-1 mutant in the presence of different ABA concentrations. Compared with WT, in which VGI was decreased by ABA, VGI of pin2-1 was completely insensitive to ABA treatment and was significantly reduced versus WT with or without ABA (Figure 7). Therefore, PIN2 was indispensable (plays a pivotal role) for the process of $\mathrm{ABA}$ root growth trajectory regulation. Alternately, $\mathrm{ABA}$ elicits the agravitropism-like phenotype by modulating the PIN2 abundance. Together with our above conclusion stating that $\mathrm{ABA}$ attenuates the SAT by decreasing the PIN2 abundance (Figure 6), these data support the deduction that ABA leads to the reduction of membrane PIN2 abundance, thereby attenuating the shootward auxin content and, consequently, the wavy root growth trajectory. 

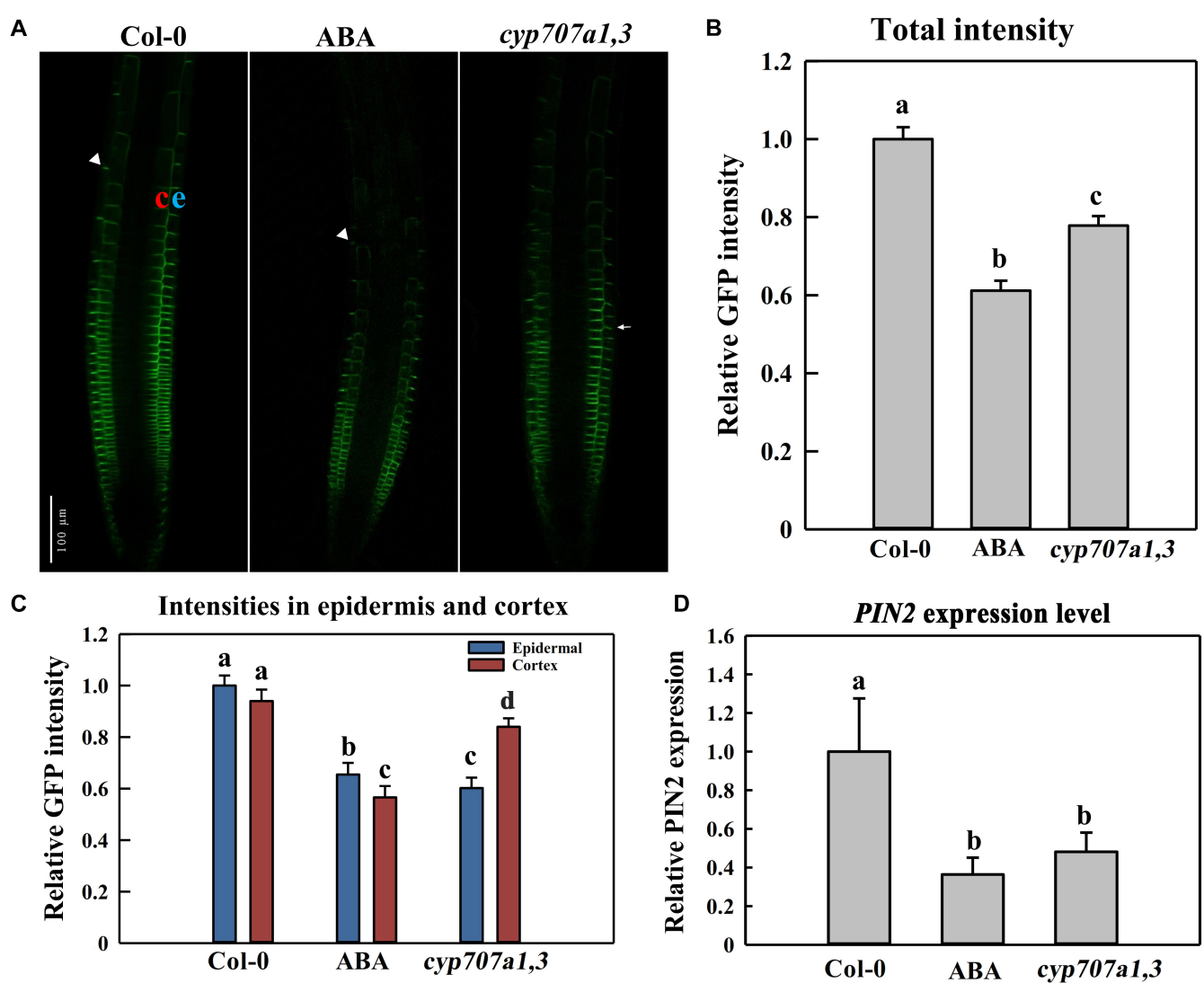

FIGURE 6 | PIN2 abundance and distribution in the control Col-0, ABA-treated WT (Col-0) and cyp707a1,3 double mutant. (A) Representative confocal images of PIN2-GFP in Col-0, ABA-treated WT and cyp707a1,3. For ABA-treated WT (middle panel), 5-day-old PIN2-GFP/Col-0 seedlings were treated with $8 \mu$ M ABA for an additional d. For Col-0 and cyp707a1,3 (left and right panels, respectively), 5-day-old seedlings were transferred onto 1/2 MS medium 1-day before confocal microscopy observations. (B) Relative total intensity of PIN2-GFP in different lines or treatments was as described in panel (A). Total PIN2-GFP intensities refer to the GFP intensity on the membrane (outlined automatically with the same threshold) and were normalized to PIN2-GFP/Col-0. (C) PIN2-GFP distribution in the epidermis and cortex of different lines or treatments was as described in panel (A). Intensities in each layer of all trials refer to the membrane intensities and were normalized to the epidermal PIN2-GFP intensity of PIN2-GFP/Col-0. (D) Relative PIN2 expression level in the root of the control Col-0, ABA-treated Col-0 and cyp707a1,3. Col-0 and ABA represent 5-day-old Col-0 seedlings treated without (Col-0) or with ABA. cyp707a1,3 was transferred onto 1/2 MS-free medium. Following 1-day treatments in the new medium, PIN2 expression level in the root tip was analyzed by qRT-PCR. The expression level was normalized to WT (Col-0). Each bar represents the mean of 3 biological samples and 3 technical replicates. For the histograms of panels (B,C), each bar represents the mean of 30 independent measurements \pm SE. For panels (B-D), the adjacent letters denote the significance levels.

The growth trajectory of a similar mutant line auxl was also tested for its response to ABA. The AUX1 is an auxin influx carrier which allows the entry of extracellular auxin into intracellular space, and is also involved in the auxin transport from root caps to the EZ (Bennett et al., 1996). Surprisingly, unlike pin2-1, the VGI of $a u x 1$ is still sensitive to ABA application (Supplementary Figure 6). The root of auxl even forms a coil upon ABA treatment. This might be due to the different auxin distribution between $a u x 1$ and pin2-1 (Swarup et al., 2001; Liu et al., 2018), although they are both involved in the SAT. Moreover, this may indicate that $\mathrm{ABA}$ regulates the root growth trajectory specifically through PIN2. Somehow, aux1 also exhibits a reduced VGI if not taking in consideration the ABA. Thus, phenotype of aux1 may ultimately correlates the impaired SAT with the agravitropism-like phenotype.

We mentioned above that the decrease in PIN2 abundance of cyp707a1,3 was mainly ascribed to its reduction in the epidermis rather than the cortex (Figure 6C). Thus, it was necessary to unravel whether the reduction in epidermal PIN2 could lead to a wavy growth trajectory. For this purpose, we expressed the PIN2-EosFP under the driven GL2 promotor in the pin2-1 mutant (Figure 8A). Because the GL2 promotor specifically expresses in the root epidermis (Lee and Schiefelbein, 1999), we obtained a partial PIN2 recovery line in which only epidermal PIN2 was expressed. We tagged this line GL2:PIN2-EosFP. Through confocal detection of the EosFP signal in GL2:PIN2-EosFP, we found that PIN2 was successfully expressed in the epidermis of this partial recovery line (Figure $\mathbf{8 B}$ ). As expected, the VGI of GL2:PIN2-EosFP was significantly higher than in pin2-1 mutant (Figures 8C,D). Thus, the epidermal PIN2 is required for controlling the root growth trajectory, and the reduction in the epidermal PIN2 abundance of cyp707a1,3 could indeed result in a reduced VGI. 


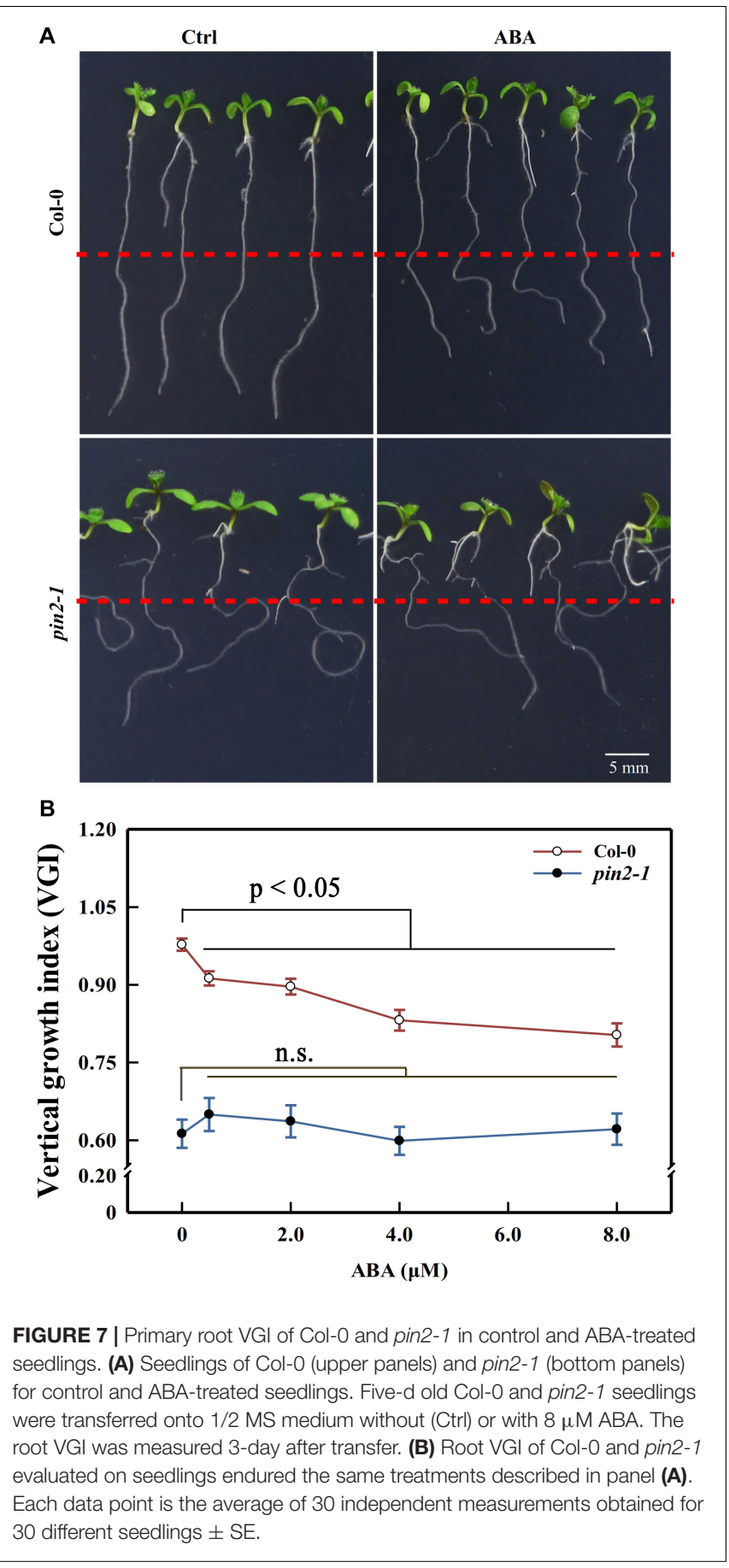

\section{ABA Reduces PIN2 Abundance Mainly Through Inhibiting Its de novo Synthesis Rather Than Accelerating Its Degradation}

The protein abundance is mainly determined by its degradation and de novo synthesis. A reduction in the PIN2 abundance might result from either its decreased synthesis or accelerated degradation. To characterize the way by which ABA reduces the root PIN2 abundance, we utilized the photoconversion fluorescent tag EosFP to segregate protein synthesis and degradation pathways. Due to the particularity of the photoconvertible material, once we implemented the photoconversion operation, the PIN2 protein already present in the membrane would be marked with red fluorescence. However, the newly synthesized PIN2 would display green color in the default PIN2EosFP state (green; Jásik et al., 2013, 2016; Wiedenmann et al., 2004). By using PIN2-EosFP, we found that the degradation rate of PIN2 was not affected either exogenously (following ABA application) or endogenously (in the cyp707a1,3 mutant) during $3 \mathrm{~h}$ after photoconversion, the RFP intensities of PIN2-EosFP in the root tip of either ABA-treated WT or cyp707a1,3 seedlings remain similar to the control: remain approximately $40 \%$ of their original intensity (Figures 9A,C). The PIN2 degradation in cyp707a1,3 seems even slightly slower than in the WT, as it possesses a higher ratio of RFP (but not significantly different). Similar result was obtained also from another transgenic line (Supplementary Figure 7). This result demonstrates that the PIN2 degradation was not accelerated by ABA.

Many membrane proteins, including PIN2, can be targeted to the lytic vacuole (LV) for degradation (Kleine-Vehn et al., 2008; Leitner et al., 2012). The acidification of the LV is prerequisite for the protein degradation. The Concanamycin A (ConcA), an inhibitor of the vacuole $\mathrm{H}^{+}$-ATPases, can reduce the acidification in LV and, thereby, inhibits the proteins degradation inside this organelle (Páli et al., 2004; Willige et al., 2011). Using $1 \mu \mathrm{M}$ ConcA, we found that the PIN2-GFP aggregation in LV was slightly inhibited upon exogenous ABA treatment (Supplementary Figures 8A,B). However, a reduced PIN2-GFP signal was detected in the LV of cyp707a1,3 (Supplementary Figure $8 \mathrm{C}$ ). These results imply that the PIN2 degradation can be inhibited by the ABA. Moreover, $\mathrm{ABA}$ induces a decrease in PIN2 LV targeting, which was not completely consistent with the results obtained by the PIN2-EosFP photoconversion, suggesting that the PIN2 degradation was probably not affected by the ABA (Figures 9A,C).

In addition, the amount of the newly synthesized PIN2 was significantly reduced by ABA, as the GFP state of PIN2-EosFP following ABA treatment or in cyp707a1,3 increased more slowly than in WT (Figures 9A,B). The relative PIN2 expression in the root tip was significantly reduced following $8 \mu \mathrm{M} \mathrm{ABA}$ treatment or in cyp707a1,3, as indicated by qRT-RCR (Figure 6D). Hence, the decrease in the amount of newly synthesized PIN2 was at least partially caused by the suppression of PIN2 expression. Thus, it is not fully clear whether the protein translation rate of PIN2 was affected. Therefore, it seems that ABA reduces the membrane PIN2 abundance mainly by reducing (slowing down) its de novo synthesis. Overall, the ABA reduces the PIN2 abundance by inhibiting its gene expression rather than accelerating of its degradation.

\section{DISCUSSION}

Considering that $\mathrm{ABA}$ is regarded as a stress plant hormone (Cutler et al., 2010; Zhang, 2014), we suppose that ABA may act 
A

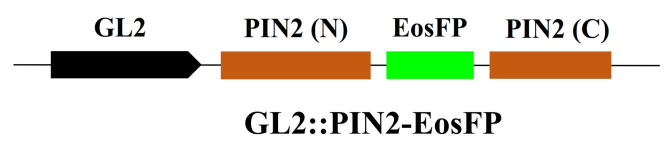

B

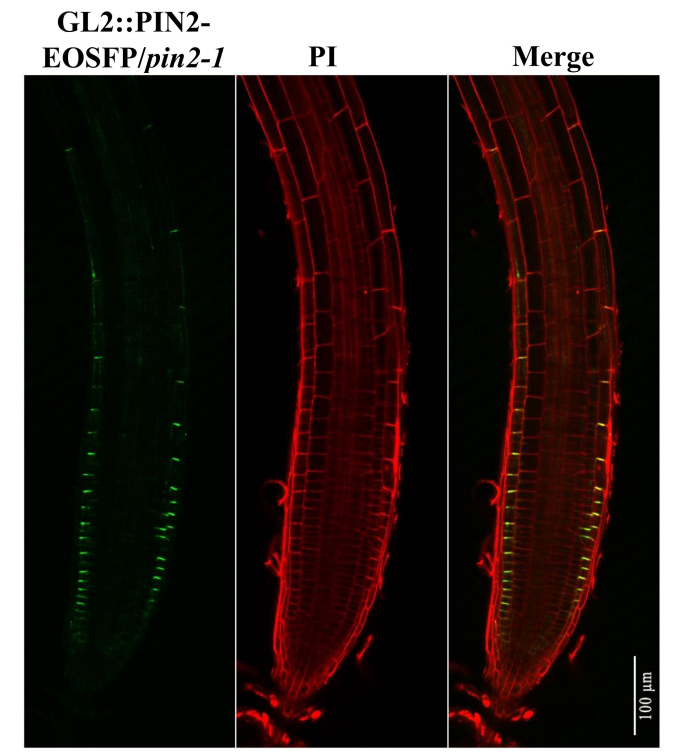

C

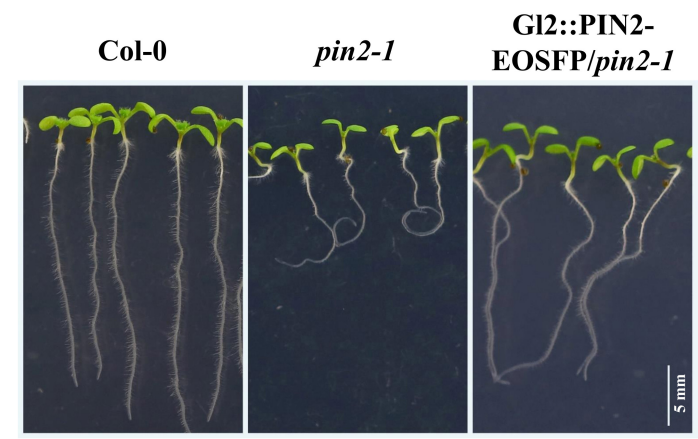

D

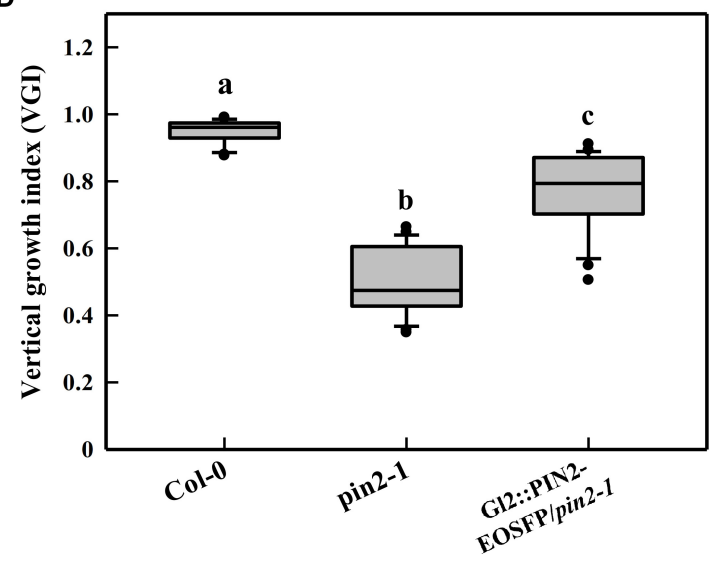

FIGURE 8 | Root VGI of the Col-0, pin2-1 and a partial recovery line GL2:PIN2-EosFP/pin2-1. (A) Schematic map of the GL2:PIN2-EosFP construct. GL2 represents the promoter of GL2, which is expressed specifically in the root epidermis. The fluorescent tag EosFP was inserted between the $\mathrm{N}$ and C-terminus of PIN2 CDS. The construct was introduced into pin2-1 mutant lines to generate a partial recovery line, GL2:PIN2-EosFP/pin2-1. (B) PIN2 expression pattern in GL2: PIN2-EosFP/pin2-1. (C) Representative seedlings of Col-0, pin2-1 and GL2:PIN2-EosFP/pin2-1. Seven-d-old seedlings are shown in the images. (D) The box plot shows the root VGI of Col-0, pin2-1 and GL2:PIN2-EosFP/pin2-1. $n>30$ for each bar.

as an intermediate between stressful conditions and root growth trajectory. Interestingly, previous researchers have shown that several adversities (including high salinity, hydrogen peroxide, soil hypoxia and bacteria) can lead to wavy root growth trajectory (Sun et al., 2008; Eysholdt-Derzso and Sauter, 2017; Zhou et al., 2018; Jimenez-Vazquez et al., 2020). This evidence clearly supports our assumption. Previously, Nie et al. (2014) investigated the characteristics of shrub roots in different soil conditions. The shrub root growth shows only a slight curvature in the soft soil but strong curvature in the hard soil (with rocks) (Nie et al., 2014). Soils with lower water content are typically harder than those with plenty of moisture. Hence, the wavy root growth trajectory in harder soil may increase the possibility of encountering moist areas with nutrients. Moreover, it has been recently reported that auxin could be involved in obstacle avoidance (Zhang and Friml, 2020). Thus, our data revealing a pivotal role for auxin in curved root growth might represent an alternative explanation for the curved root phenotype induced by hard soil-roots would apparently encounter obstacles more frequently in hard soil.

Previously, Sun et al. (2008) had reported the same phenotype induced by a high level of $\mathrm{NaCl}$. Authors ascribed the curved phenotype to a compromised gravitropism. The tropic growth to other stimuli would become visible with the overwhelming decrease in the gravitropism (Sun et al., 2008). Interestingly, the parameter VGI that we used to quantify root curvature was suggested as a sensitive morphometric parameter to assess the defect in gravitropism (Grabov et al., 2005; Rigas et al., 2013; Wang et al., 2013). However, our results indicate that the agravitropism-like phenotype did not result from the compromised gravitropism. In our context, the gravitropic bending rate was not affected by $2 \mu \mathrm{M}$ ABA (Supplementary Figure 9). However, this $A B A$ concentration was sufficient to elicit the agravitropism-like phenotype (Figure 1). Consistently, several reports documented that ABA is unlikely to be a regulator of the root gravitropism (Lee et al., 1990; Moore, 1990; Young and Evans, 1996). Hence, we suggest that VGI reveals a different physiological aspect of the gravitropism reflected by gravitropic bending. VGI seems to be a long-term reaction or modulation of gravitropism that may last for several days. However, the gravitropic bending is usually completed within a maximum of 24 h (Hu et al., 2005; Perera et al., 2006).

The crosstalk between ABA and auxin in the context of root growth were under extensive investigations. For examples: 

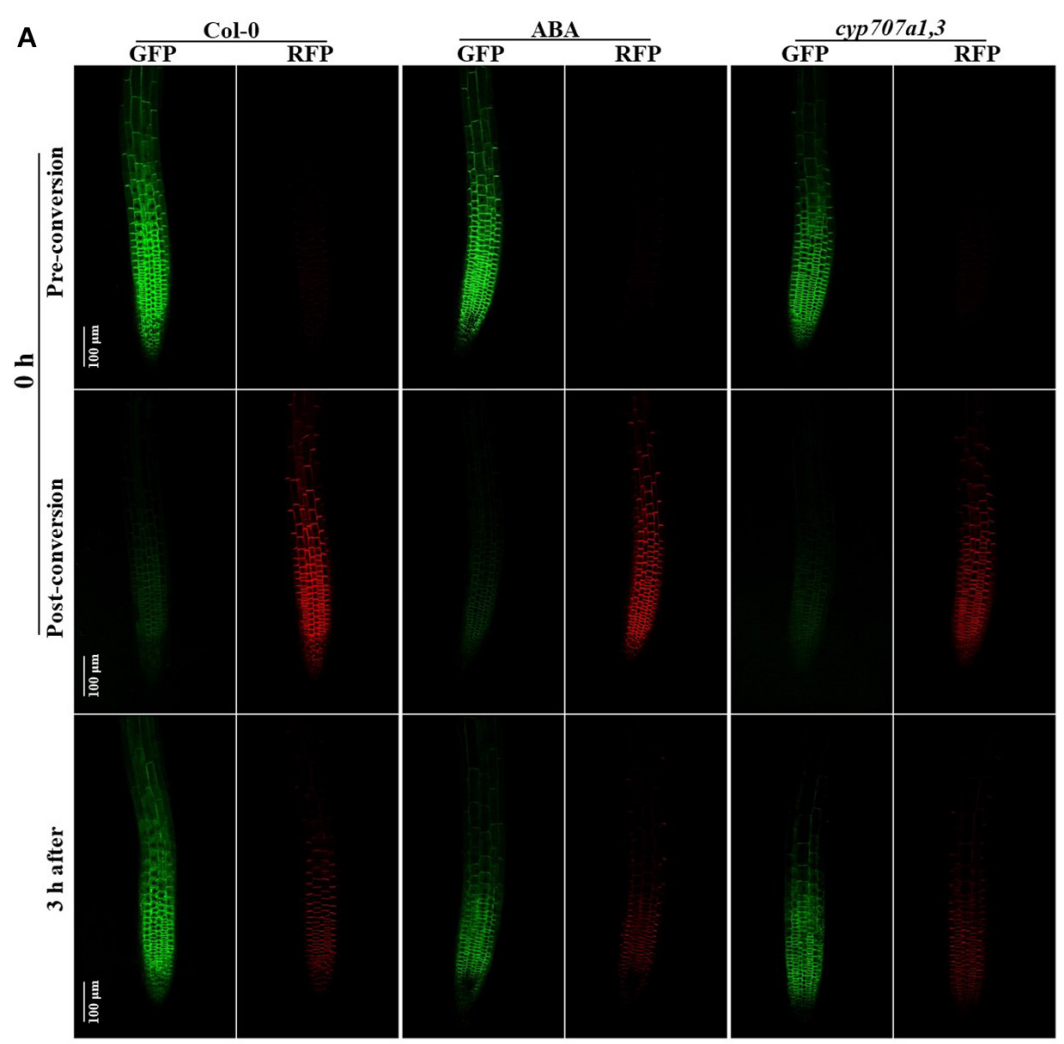

B

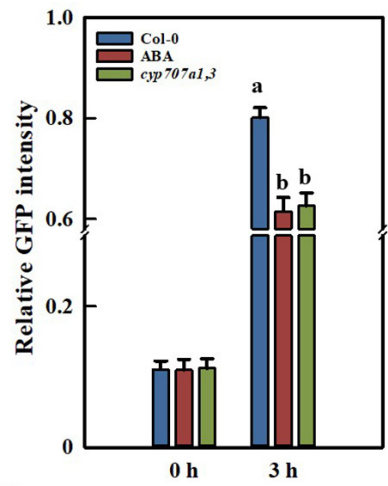

C

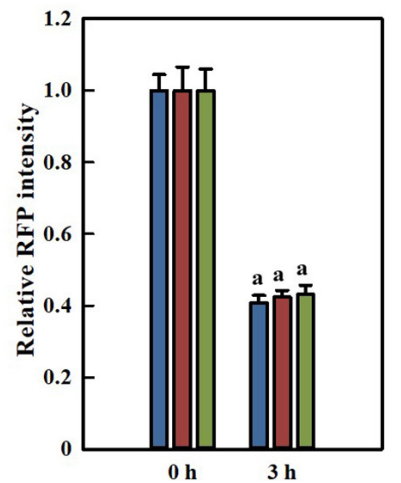

FIGURE 9 | PIN2 synthesis and degradation in Control Col-0, ABA-treated Col-0 and the cyp707a1,3 double mutant as revealed by the photoconversion of PIN2-EosFP. (A) Representative confocal images of PIN2-EosFP (including GFP and RFP) in Col-0, ABA-treated Col-0 and cyp707a1,3. The 6 upper panels show the pre-conversion images of PIN2-EosFP (GFP state) that was not yet converted into RFP. After a 30-s exposure to 405-nm irradiation, EosFP was converted to the post-conversion state in which most PIN2-EosFP was in the RFP state (middle panels). Three hours after conversion, the dynamics of the GFP and RFP signals (bottom panels) were measured to evaluate the rate of PIN2 synthesis and degradation. Five-d-old seedlings were used. After photoconversion, the PIN2-EosFP/Col-0 seedlings were directly transferred onto 1/2 MS medium with or without $8 \mu \mathrm{M}$ ABA, and they were labeled Col-0 (left panels column) and ABA (middle panels column). For the PIN2-EosFP/cyp707a1,3 seedlings were also transferred onto 1/2 MS after photoconversion (right panels column). (B) Increasing speed of GFP within $3 \mathrm{~h}$. Col-0, ABA and cyp707a1,3 seedlings were treated as described in panel (A). Relative intensities of Col-0, ABA and cyp707a1,3 refer to the membrane intensities normalized to their original GFP intensities (pre-conversion). (C) Decreasing rate of RFP within $3 \mathrm{~h}$. Same figure legend as in panel (B). For the histograms of panels $(\mathbf{B}, \mathbf{C})$, each bar is the average of 30 independent measurements made on different roots \pm SE. The relative intensities of Col-0, $\mathrm{ABA}$ and cyp707a1,3 represent the membrane intensities normalized to their original RFP intensities (post-conversion).

Munguia-Rodriguez et al. (2020) proved that excess auxin content may confer oversensitivity to root elongation induced by ABA; Thole et al. (2014) had demonstrated that root elongation in both aux1 and pin2 is insensitive to ABA. These together demonstrated that ABA could affect root growth through modulating auxin transport, and is in line with our main conclusions that $\mathrm{ABA}$ regulates root growth trajectory via auxin transporter (PIN2; Figure 10). The above cited researches shade lights on the correlations between hormonal crosstalk (ABA and auxin) and root elongation. Our work is dedicated to studying the role of hormonal crosstalk in root growth trajectory. We provide evidence that $\mathrm{ABA}$ regulates the root growth trajectory/direction by modulating PIN2 abundance (Figure 5). Considering the versatile function of $\mathrm{ABA}$ in the stress response, our results indicate that PIN2 may serve as an intermedia between the stress conditions and root growth trajectory. Consistently, the role of PIN2 in growth adaptability has been emphasized
(Remy et al., 2013). Moreover, we demonstrate the importance of epidermal PIN2 in modulating the root growth trajectory (Figure 6), which had never been previously documented.

We notice that the activity of the GL2 promoter was much weaker than the PIN2 native promoter because even in the strongest line selected among 20 independent GL2:PIN2-EosFP/pin2-1 $\mathrm{T}_{3}$ lines according to their PIN2 expression, the PIN2-EosFP intensity was much weaker than the average recorded in the epidermis of PIN2:PIN2EosFP/pin2-1. However, even driven by a frail promotor, GL2:PIN2-EosFP shows a substantial increase in VGI from 0.49 to 0.77 , representing an approximately $60 \%$ difference between pin2-1 and WT (Figure 8). Thus, the epidermal PIN2 bears (assumes) a major responsibility in controlling the root trajectory/direction. This is a plausible revelation because the epidermis is located in the root outermost layer. Accordingly, such physiological processes, including asymmetric 


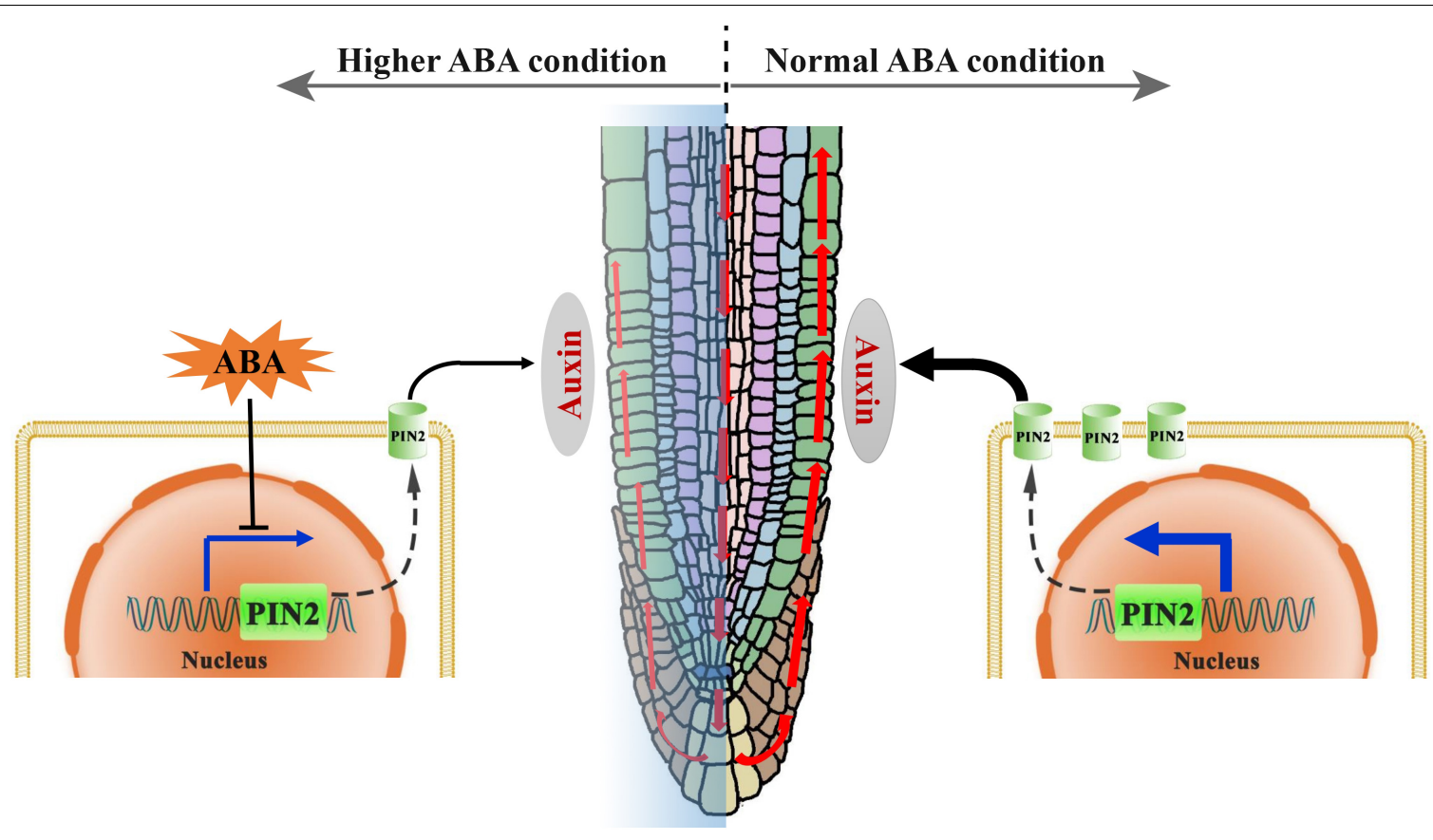

FIGURE 10 | A proposed mechanistic model for the regulation of root growth trajectory in response to ABA. In normal (ambient) environmental condition, the auxin can be transported from the lateral root caps toward the elongation zone (EZ), mainly through a polar distributed auxin efflux carrier PIN2 (green cylinder in the membrane). A certain level of auxin in the $\mathrm{EZ}$ is required for the maintaining of a symmetric cell elongation. However, unfavorable environmental conditions could induce an elevated ABA level in root. Hence, higher ABA level suppresses the PIN2 transcription resulting thus in a lower PIN2 abundance, and leads consequently to the impairment of SAT (red upward arrowheads). An insufficiency in the auxin content in the EZ causes ultimately to an asymmetric cell elongation in this region, conferring thereby a wavy growth trajectory.

cell elongation during tropic bending, would certainly be exacerbated in the epidermis.

We noticed that after transfer, the lateral roots growth was apparently promoted by $\mathrm{ABA}$, since both of the lateral roots number and length were increased in the $1 / 2 \mathrm{MS}$ medium containing ABA (Figure 1 and Supplementary Figure 10). Recently, Jimenez-Vazquez et al. (2020) have reported that a beneficial rhizobacterium Achromobacter sp. 5B1 could elicit a similar phenotype as ABA does. Interestingly, the bacteria also promotes lateral root formation which may due to increased wavy frequency along growth trajectory (Jimenez-Vazquez et al., 2020). Although ABA promotes lateral roots elongation and formation as well as the wavy amplitude of growth trajectory (Figure 1). Meanwhile, promotion on lateral roots does not occur in the curved part of the root (a root part grown after transfer). Therefore, the mechanism underlines the ABA promoting effect on the lateral root formation might be different from that reported by Jimenez-Vazquez et al. (2020).

In conclusion, we revealed in this report that the exogenous and endogenous ABA accumulation could result in the root agravitropism-like growth pattern. The agravitropism-like phenotype resulted from the reduced auxin content of SAT caused by ABA (Figure 10). ABA mitigation of the SAT by decreasing the PIN2 abundance, and the latter (reduction in PIN2 abundance) was attributable to the suppression of its gene expression rather than the acceleration of its degradation (Figure 10). Altogether, ABA suppresses the PIN2 expression, inhibiting thereby the SAT (Figure 10). This attenuation of SAT would ultimately elicit the agravitropism-like phenotype.

\section{DATA AVAILABILITY STATEMENT}

The original contributions presented in the study are included in the article/Supplementary Material, further inquiries can be directed to the corresponding author/s.

\section{AUTHOR CONTRIBUTIONS}

QX and WC: conceptualization and writing. QX, WC, and JE: methodology and design. QX, JE, XP, and HC: experiments. $\mathrm{QX}, \mathrm{XP}$, and HC: investigation. WC: funding acquisition and supervision. QX and JE: resources. All authors contributed to the article and approved the submitted version.

\section{FUNDING}

This work was supported by the national natural science foundation of China, NSFC (Grant Nos. 31570859, 31971172, and U1738107), the strategic priority research program of the Chinese Academy of Sciences, CAS (Grant Nos. XDA0402020215 and XDA04020415), and the China manned space flight technology project. 


\section{ACKNOWLEDGMENTS}

We thank Dr. Sheng Teng (institute of plant physiology and ecology, Shanghai, China), for providing us the cyp707a1,3 double mutant. Thanks also to Dr. Huiqiong Zheng (institute of plant physiology and ecology, Shanghai, China) for kindly gift us the pin2-1 mutant. We thank Dr. Yuanhong Shan and Dr. Yining Liu in the core Facility Centre of Institute of Plant Physiology and Ecology for mass spectrometry assistance. We also

\section{REFERENCES}

Ackerson, R. C. (1984). Abscisic acid and precocious germination in soybeans. J. Exp. Bot. 35, 414-421. doi: 10.1093/jxb/35.3.414

Benech-Arnold, R. L., Gualano, N., Leymarie, J., Côme, D., and Corbineau, F. (2006). Hypoxia interferes with ABA metabolism and increases ABA sensitivity in embryos of dormant barley grains. J. Exp. Bot. 57, 1423-1430. doi: 10.1093/ jxb/erj122

Benková, E., Michniewicz, M., Sauer, M., Teichmann, T., Seifertová, D., Jürgens, G., et al. (2003). Local, Efflux-Dependent Auxin Gradients as a Common Module for Plant Organ Formation. Cell 115, 591-602.

Bennett, M. J., Marchant, A., Green, H. G., May, S. T., Ward, S. P., Millner, P. A., et al. (1996). Arabidopsis AUX1 gene: a permease-like regulator of root gravitropism. Science 273, 948-950.

Birnbaum, K., Shasha, D. E., Wang, J. Y., Jung, J. W., Lambert, G. M., Galbraith, D. W., et al. (2003). A gene expression map of the Arabidopsis root. Science 302, 1956-1960.

Bourgeois, D., Regis-Faro, A., and Adam, V. (2012). Photoactivated structural dynamics of fluorescent proteins. Biochem. Soc. Trans. 40, 531-538. doi: 10. 1042/BST20120002

Cassab, G. I., Eapen, D., and Campos, M. E. (2013). Root hydrotropism: an update. Am. J. Bot. 100, 14-24. doi: 10.3732/ajb.1200306

Chen, R., Hilson, P., Sedbrook, J., Rosen, E., Caspar, T., and Masson, P. H. (1998). The Arabidopsis thaliana AGRAVITROPIC1 gene encodes a component of the polar-auxin-transport efflux carrier. P. Natl. Acad. Sci. U S A. 95, $15112-15117$

Cutler, S. R., Rodriguez, P. L., Finkelstein, R. R., and Abrams, S. R. (2010). Abscisic acid: emergence of a core signaling network. Ann. Rev. Plant Bio. 61, 651-679.

Dhonukshe, P., Aniento, F., Hwang, I., Robinson, D. G., Mravec, J., Stierhof, Y.D., et al. (2007). Clathrin-mediated constitutive endocytosis of PIN auxin efflux carriers in Arabidopsis. Curr. Biol. 17, 520-527. doi: 10.1016/j.cub.2007.01.052

Eysholdt-Derzso, E., and Sauter, M. (2017). Root bending is antagonistically affected by hypoxia and erf-mediated transcription via auxin signaling. Plant Physiol. 175, 412-423. doi: 10.1104/pp.17.00555

Finkelstein, R. R., Gampala, S. S., and Rock, R. D. (2002). Abscisic acid signaling in seeds and seedlings. Plant Cell 14 Suppl(Suppl.), S15-S45.

Friml, J. (2003). Auxin transport - shaping the plant. Curr. Opin. Plant Biol. 6, 7-12. doi: 10.1016/S1369526602000031

Grabov, A., Ashley, M. K., Rigas, S., Hatzopoulos, P., Dolan, L., and Vicente-Agullo, F. (2005). Morphometric analysis of root shape. N. Phytol. 165, 641-651.

Heisler, M. G., Ohno, C., Das, P., Sieber, P., Reddy, G. V., Long, J. A., et al. (2005). Patterns of auxin transport and gene expression during primordium development revealed by live imaging of the Arabidopsis inflorescence meristem. Curr. Biol. 15, 1899-1911.

Hu, X., Neill, S. J., Tang, Z., and Cai, W. (2005). Nitric oxide mediates gravitropic bending in soybean roots. Plant Physiol. 137, 663-670. doi: 10.1104/pp.104. 054494

Hung, K. T., and Kao, C. H. (2004). Hydrogen peroxide is necessary for abscisic acid-induced senescence of rice leaves. Plant Physiol. 161, 1347-1357. doi: 10. 1016/j.jplph.2004.05.011

Jásik, J., Boggetti, B., Baluška, F., Volkmann, D., Gensch, T., Rutten, T., et al. (2013). PIN2 turnover in Arabidopsis root epidermal cells explored by the photoconvertible protein Dendra2. PLoS One 8:e61403. doi: 10.1371/journal. pone.0061403 thank Professor Feng Yu (Hunan university) for his help on experimental improvement and suggestions on manuscript writing.

\section{SUPPLEMENTARY MATERIAL}

The Supplementary Material for this article can be found online at: https://www.frontiersin.org/articles/10.3389/fpls.2021. 632676/full\#supplementary-material

Jásik, J., Bokor, B., Stuchlík, S., Mičieta, K., Turňa, J., and Schmelzer, E. (2016) Effects of auxins on PIN-FORMED2 (PIN2) dynamics are not mediated by inhibiting PIN2 endocytosis. Plant Physiol. 172, 1019-1031.

Jimenez-Vazquez, K. R., Garcia-Cardenas, E., Barrera-Ortiz, S., Ortiz-Castro, R., Ruiz-Herrera, L. F., Ramos-Acosta, B. P., et al. (2020). The plant beneficial rhizobacterium Achromobacter sp. 5B1 influences root development through auxin signaling and redistribution. Plant J. 103, 1639-1654. doi: 10.1111/tpj. 14853

Kleine-Vehn, J., Dhonukshe, P., Sauer, M., Brewer, P. B., Wiśniewska, J., Paciorek, T., et al. (2008). ARF GEF-dependent transcytosis and polar delivery of PIN auxin carriers in Arabidopsis. Curr. Biol. 18, 526-531.

Kramer, E. M., and Bennett, M. J. (2006). Auxin transport: a field in flux. Trends Plant Sci. 11, 382-386. doi: 10.1016/j.tplants.2006.06.002

Lee, H. J., Kim, H. S., Park, J. M., Cho, H. S., and Jeon, J. H. (2020). PINmediated polar auxin transport facilitates root-obstacle avoidance. N. Phytol. 225, 1285-1296. doi: 10.1111/nph.16076

Lee, J. S., Hasenstein, K.-H., Mulkey, T. J., Yang, R. L., and Evans, M. L. (1990) Effects of abscisic acid and xanthoxin on elongation and gravitropism in primary roots of zea mays. Plant Sci. 68, 17-26. doi: 10.1016/0168-9452(90) 90147-g

Lee, M. M., and Schiefelbein, J. (1999). WEREWOLF, a MYB-Related Protein in Arabidopsis, Is a Position-Dependent Regulator of Epidermal Cell Patterning. Cell 99, 473-483. doi: 10.1016/S0092-8674(00)81536-6

Leitner, J., Retzer, K., Korbei, B., and Luschnig, C. (2012). Dynamics in PIN2 auxin carrier ubiquitylation in gravity-responding Arabidopsis roots. Plant Signal Behav. 7, 1271-1273. doi: 10.4161/psb.21715

Lippincott-Schwartz, J., and Patterson, G. H. (2008). Fluorescent proteins for photoactivation experiments. Methods Cell Biol. 85, 45-61. doi: 10.1016/S0091679X(08)85003-0

Liu, H., Liu, B., Chen, X., Zhu, H., Zou, C., and Men, S. (2018). AUX1 acts upstream of PIN2 in regulating root gravitropism. Biochem. Biophys. Res. Commun. 507, 433-436.

Liu, X., Zhang, H., Zhao, Y., Feng, Z., Li, Q., Yang, H. Q., et al. (2013). Auxin controls seed dormancy through stimulation of abscisic acid signaling by inducing ARF-mediated ABI3 activation in Arabidopsis. Proc. Natl. Acad. Sci. U.S.A 110, 15485-15490. doi: 10.1073/pnas.130465 1110

Livak, K. J., and Schmittgen, T. D. (2001). Analysis of relative gene expression data using real-time quantitative PCR and the 2(-Delta Delta C(T)) Method. Methods 25, 402-408. doi: 10.1006/meth.2001.1262

Marchant, A., Kargul, J., May, S. T., Muller, P., Delbarre, A., Perrot-Rechenmann, C., et al. (1999). AUX1 regulates root gravitropism in Arabidopsis by facilitating auxin uptake within root apical tissues. EMBO J. 18, 2066-2073. doi: 10.1093/ emboj/18.8.2066

Márquez-López, R. E., Ku-González, Á, Méndez-Hernández, H. A., Galaz-Ávalos, R. M., and Loyola-Vargas, V. M. (2018). Auxin immunolocalization in Coffea canephora Tissues. Methods Mol. Biol. 1815, 179-188.

Moore, R. (1990). Abscisic acid is not necessary for gravitropism in primary roots of zea mays. Ann. Bot. 66, 281-283. doi: 10.1093/oxfordjournals.aob.a08 8026

Mullen, J. L., Turk, E., Johnson, K., Wolverton, C., Ishikawa, H., Simmons, C., et al. (1998). Root-growth behavior of the Arabidopsis Mutantrgr1: roles of gravitropism and circumnutating in the waving/coiling phenomenon. Plant Physiol. 118, 1139-1145. 
Müller, A., Guan, C., Gälweiler, L., Tänzler, P., Huijser, P., Marchant, A., et al. (1998). AtPIN2 defines a locus of Arabidopsis for root gravitropism control. ЕМВО J. 17, 6903-6911.

Munguia-Rodriguez, A. G., Lopez-Bucio, J. S., Ruiz-Herrera, L. F., Ortiz-Castro, R., Guevara-Garcia, A. A., Marsch-Martinez, N., et al. (2020). YUCCA4 overexpression modulates auxin biosynthesis and transport and influences plant growth and development via crosstalk with abscisic acid in Arabidopsis thaliana. Genet. Mol. Biol. 43:e20190221. doi: 10.1590/1678-4685-gmb-20190221

Neuman, D. S., and Smit, B. A. (1991). The influence of leaf water status and aba on leaf growth and stomata of phaseolus seedlings with hypoxic roots. J. Exp. Bot. 42, 1499-1506. doi: 10.1093/jxb/42.12.1499

Nie, Y. P., Chen, H. S., Wang, K. L., and Ding, Y. L. (2014). Rooting characteristics of two widely distributed woody plant species growing in different karst habitats of southwest China. Plant Ecol. 215, 1099-1109. doi: 10.1007/s11258-0140369-0

Okamoto, M., Kuwahara, A., Seo, M., Kushiro, T., Asami, T., Hirai, N., et al. (2006). CYP707A1 and CYP707A2, which encode abscisic acid 8'-hydroxylases, are indispensable for proper control of seed dormancy and germination in Arabidopsis. Plant Physiol. 141, 97-107. doi: 10.1104/pp.106.079475

Páli, T., Dixon, N., Kee, T. P., and Marsh, D. (2004). Incorporation of the V-ATPase inhibitors concanamycin and indole pentadiene in lipid membranes. Spin-label EPR studies. Biochim. Biophys. Acta 1663, 14-18. doi: 10.1016/j.bbamem.2004. 03.003

Pan, X., Welti, R., and Wang, X. (2010). Quantitative analysis of major plant hormones in crude plant extracts by high-performance liquid chromatographymass spectrometry. Nat. Protoc. 5, 986-992. doi: 10.1038/nprot.2010.37

Pasternak, T., Tietz, O., Rapp, K., Begheldo, M., Nitschke, R., Ruperti, B., et al. (2015). Protocol: an improved and universal procedure for whole-mount immunolocalization in plants. Plant methods 11:50. doi: 10.1186/s13007-0150094-2

Pei, Z. M., Murata, Y., Benning, G., Thomine, S., Klusener, B., Allen, G. J., et al. (2000). Calcium channels activated by hydrogen peroxide mediate abscisic acid signalling in guard cells. Nature 406, 731-734.

Perera, I. Y., Hung, C. Y., Brady, S., Muday, G. K., and Boss, W. F. (2006). A universal role for inositol 1,4,5-trisphosphate-mediated signaling in plant gravitropism. Plant Physiol. 140, 746-760. doi: 10.1104/pp.105.075119

Postma, J. A., Dathe, A., and Lynch, J. P. (2014). The optimal lateral root branching density for maize depends on nitrogen and phosphorus availability. Plant Physiol. 166, 590-602. doi: 10.1104/pp.113.233916

Qi, B., and Zheng, H. (2013). Modulation of root-skewing responses by KNAT1 in Arabidopsis thaliana. Plant J. 76, 380-392. doi: 10.1111/tpj.12295

Remy, E., Cabrito, T. R., Baster, P., Batista, R. A., Teixeira, M. C., Friml, J., et al. (2013). A major facilitator superfamily transporter plays a dual role in polar auxin transport and drought stress tolerance in Arabidopsis. Plant Cell 25, 901-926. doi: 10.1105/tpc.113.110353

Rigas, S., Ditengou, F. A., Ljung, K., Daras, G., Tietz, O., Palme, K., et al. (2013). Root gravitropism and root hair development constitute coupled developmental responses regulated by auxin homeostasis in the Arabidopsis root apex. N. Phytol. 197, 1130-1141. doi: 10.1111/nph.12092

Roy, R., and Bassham, D. C. (2014). Root growth movements: waving and skewing. Plant Sci. 221, 42-47. doi: 10.1016/j.plantsci.2014.01.007

Ruegger, M., Dewey, E., Gray, W. M., Hobbie, L., Turner, J., and Estelle, M. (1998). The TIR1 protein of Arabidopsis functions in auxin response and is related to human SKP2 and yeast grrlp. Genes Dev. 12, 198-207.

Sato, A., Sasaki, S., Matsuzaki, J., and Yamamoto, K. T. (2014). Light-dependent gravitropism and negative phototropism of inflorescence stems in a dominant Aux/IAA mutant of Arabidopsis thaliana, axr2. J. Plant Res. 127, 627-639. doi: 10.1007/s10265-014-0643-1

Shin, H., Shin, H.-S., Guo, Z., Blancaflor, E. B., Masson, P. H., and Chen, R. (2005). Complex regulation of Arabidopsis AGR1/PIN2-mediated root gravitropic response and basipetal auxin transport by cantharidin-sensitive protein phosphatases. Plant J. 42, 188-200. doi: 10.1111/j.1365-313X.2005. 02369.x

Su, S. H., Gibbs, N. M., Jancewicz, A. L., and Masson, P. H. (2017). Molecular mechanisms of root gravitropism. Curr. Biol. 27, R964-R972.

Sun, F., Zhang, W., Hu, H., Li, B., Wang, Y., Zhao, Y., et al. (2008). Salt modulates gravity signaling pathway to regulate growth direction of primary roots in Arabidopsis. Plant Physiol. 146, 178-188.
Swarup, R., Friml, J., Marchant, A., Ljung, K., Sandberg, G., Palme, K., et al. (2001). Localization of the auxin permease AUX1 suggests two functionally distinct hormone transport pathways operate in the Arabidopsis root apex. Genes Dev. 15, 2648-2653. doi: 10.1101/gad.210501

Thole, J. M., Beisner, E. R., Liu, J., Venkova, S. V., and Strader, L. C. (2014). Abscisic acid regulates root elongation through the activities of auxin and ethylene in Arabidopsis thaliana. G3 4, 1259-1274.

Veselov, D. S., Sharipova, G. V., Veselov, S. U., and Kudoyarova, G. R. (2008). The effects of nacl treatment on water relations, growth, and aba content in barley cultivars differing in drought tolerance. J. Plant Growth Regul. 27, 380-386. doi: 10.1007/s00344-008-9064-5

Walch-Liu, P., Ivanov, I. I., Filleur, S., Gan, Y., Remans, T., and Forde, B. G. (2006). Nitrogen regulation of root branching. Ann. Bot. 97, 875-881.

Wang, C., Yan, X., Chen, Q., Jiang, N., Fu, W., Ma, B., et al. (2013). Clathrin light chains regulate clathrin-mediated trafficking, auxin signaling, and development in Arabidopsis. Plant Cell 25, 499-516.

Weng, L., Zhao, F., Li, R., Xu, C., Chen, K., and Xiao, H. (2015). The zinc finger transcription factor SIZFP2 negatively regulates abscisic acid biosynthesis and fruit ripening in tomato. Plant Physiol. 167, 931-949.

Wiedenmann, J., Ivanchenko, S., Oswald, F., Schmitt, F., Röcker, C., Salih, A., et al. (2004). EosFP, a fluorescent marker protein with UV-inducible green-to-red fluorescence conversion. P. Natl. Acad. Sci. U S A. 101, 15905-15910.

Willige, B. C., Isono, E., Richter, R., Zourelidou, M., and Schwechheimer, C. (2011). Gibberellin regulates PIN-FORMED abundance and is required for auxin transport-dependent growth and development in Arabidopsis thaliana. Plant Cell 23, 2184-2195. doi: 10.1105/tpc.111.086355

Winter, D., Vinegar, B., Nahal, H., Ammar, R., Wilson, G. V., and Provart, N. J. (2007). An "Electronic Fluorescent Pictograph" browser for exploring and analyzing large-scale biological data sets. PLoS One 2:e718. doi: 10.1371/journal. pone.0000718

Xu, W., Jia, L., Shi, W., Liang, J., Zhou, F., Li, Q., et al. (2013). Abscisic acid accumulation modulates auxin transport in the root tip to enhance proton secretion for maintaining root growth under moderate water stress. N. Phytol. 197, 139-150. doi: 10.1111/nph.12004

Yano, R., Kanno, Y., Jikumaru, Y., Nakabayashi, K., Kamiya, Y., and Nambara, E. (2009). CHOTTO1, a putative double APETALA2 repeat transcription factor, is involved in abscisic acid-mediated repression of gibberellin biosynthesis during seed germination in Arabidopsis. Plant Physiol. 151, 641-654. doi: 10.1104/pp. 109.142018

Yoshida, T., Fujita, Y., Maruyama, K., Mogami, J., Todaka, D., Shinozaki, K., et al. (2015). Four Arabidopsis AREB/ABF transcription factors function predominantly in gene expression downstream of SnRK2 kinases in abscisic acid signalling in response to osmotic stress. Plant Cell Environ. 38, 35-49. doi: $10.1111 /$ pce. 12351

Young, L. M., and Evans, M. L. (1996). Patterns of auxin and abscisic acid movement in the tips of gravistimulated primary roots of maize. Plant Growth Regul. 20, 253-258. doi: 10.1007/BF00043315

Zhang, D. (2014). Abscisic Acid: Metabolism, Transport and Signaling. New York, NY: Springer. doi: 10.1007/978-94-017-9424-4

Zhang, Y., and Friml, J. (2020). Auxin guides roots to avoid obstacles during gravitropic growth. N. Phytol. 225:1049. doi: 10.1111/nph. 16203

Zhou, L., Hou, H., Yang, T., Lian, Y., Sun, Y., Bian, Z., et al. (2018). Exogenous hydrogen peroxide inhibits primary root gravitropism by regulating auxin distribution during Arabidopsis seed germination. Plant Physiol. Biochem. 128, $126-133$.

Conflict of Interest: The authors declare that the research was conducted in the absence of any commercial or financial relationships that could be construed as a potential conflict of interest.

Copyright (c) 2021 Xie, Essemine, Pang, Chen, Jin and Cai. This is an open-access article distributed under the terms of the Creative Commons Attribution License (CC BY). The use, distribution or reproduction in other forums is permitted, provided the original author(s) and the copyright owner(s) are credited and that the original publication in this journal is cited, in accordance with accepted academic practice. No use, distribution or reproduction is permitted which does not comply with these terms. 